Article

\title{
Lysosomal Function Impacts the Skeletal Muscle Extracellular Matrix
}

\author{
Elizabeth C. Coffey ${ }^{1,+} \oplus^{\mathbb{C}}$, Mary Astumian ${ }^{2}\left(\mathbb{D}\right.$, Sarah S. Alrowaished ${ }^{1, \ddagger}$, Claire Schaffer ${ }^{1}$ \\ and Clarissa A. Henry $1,2, *$ (D) \\ 1 School of Biology and Ecology, University of Maine, Orono, ME 04469, USA; \\ Elizabeth.Coffey@stjude.org (E.C.C.); salrowaished@kfu.edu.sa (S.S.A.); claire.Schaffer@maine.edu (C.S.) \\ 2 Graduate School of Biomedical Science and Engineering, University of Maine, Orono, ME 04469, USA; \\ Mary.Astumian@maine.edu \\ * Correspondence: clarissa.henry@maine.edu; Tel.: +1-207-581-2816 \\ † Current location: St. Jude Children's Research Hospital, Memphis, TN 38105, USA. \\ $\ddagger$ Current location: Department of Biological Sciences, King Faisal University, Al Hofuf 31982, Saudi Arabia.
}

\section{check for} updates

Citation: Coffey, E.C.; Astumian, M.; Alrowaished, S.S.; Schaffer, C.; Henry, C.A. Lysosomal Function Impacts the Skeletal Muscle Extracellular Matrix. J. Dev. Biol. 2021, 9, 52. https:// doi.org/10.3390/jdb9040052

Academic Editors: Rosa Serra and Simon J Conway

Received: 24 September 2021

Accepted: 13 November 2021

Published: 23 November 2021

Publisher's Note: MDPI stays neutral with regard to jurisdictional claims in published maps and institutional affiliations.

Copyright: (c) 2021 by the authors. Licensee MDPI, Basel, Switzerland. This article is an open access article distributed under the terms and conditions of the Creative Commons Attribution (CC BY) license (https:// creativecommons.org/licenses/by/ $4.0 /)$.

\begin{abstract}
Muscle development and homeostasis are critical for normal muscle function. A key aspect of muscle physiology during development, growth, and homeostasis is modulation of protein turnover, the balance between synthesis and degradation of muscle proteins. Protein degradation depends upon lysosomal $\mathrm{pH}$, generated and maintained by proton pumps. Sphingolipid transporter 1 (spns1), a highly conserved gene encoding a putative late endosome/lysosome carbohydrate $/ \mathrm{H}^{+}$ symporter, plays a pivotal role in maintaining optimal lysosomal $\mathrm{pH}$ and $\mathrm{spns} 1^{-/-}$mutants undergo premature senescence. However, the impact of dysregulated lysosomal $\mathrm{pH}$ on muscle development and homeostasis is not well understood. We found that muscle development proceeds normally in spns $1^{-/-}$mutants prior to the onset of muscle degeneration. Dysregulation of the extracellular matrix (ECM) at the myotendinous junction (MTJ) coincided with the onset of muscle degeneration in spns1 $1^{-/-}$mutants. Expression of the ECM proteins laminin 111 and MMP-9 was upregulated. Upregulation of laminin 111 mitigated the severity of muscle degeneration, as inhibition of adhesion to laminin 111 exacerbated muscle degeneration in spns $1^{-/-}$mutants. MMP-9 upregulation was induced by tnfsf12 signaling, but abrogation of MMP-9 did not impact muscle degeneration in spns1 $1^{-/}$mutants. Taken together, these data indicate that dysregulated lysosomal $\mathrm{pH}$ impacts expression of ECM proteins at the myotendinous junction.
\end{abstract}

Keywords: zebrafish; spinster; lysosomal myopathy; basement membrane; myotendinous junction; skeletal muscle

\section{Introduction}

Proper development and maintenance of skeletal muscle is essential for normal physiology and locomotion. Protein turnover plays a major role in muscle development and homeostasis. For example, artificial selection of chicken lines for fast and slow growth rates suggests that the rate of protein degradation is the major factor underlying overall muscle growth during development in amniotes [1]. Additionally, protein degradation has been shown to underlie muscle growth in most bony fish [2]. Protein degradation mainly takes place in the lysosome, a principal proteolytic system that accounts for more than $98 \%$ of long-lived bulk protein turnover in skeletal muscle [3]. The efficiency of lysosomal protein degradation depends upon acidic lysosomal $\mathrm{pH}$. This dependence is because lysosomal protein degradation requires cathepsins that are optimally active at a $\mathrm{pH}$ of $4.5-5$. Lysoso$\mathrm{mal} \mathrm{pH}$ is generated and maintained by lysosomal proton pumps. Despite the importance of protein degradation in muscle growth during development, the impact of dysregulated lysosomal $\mathrm{pH}$ on muscle development, growth, and homeostasis is unknown.

Adhesion of muscle fibers to their surrounding extracellular matrix (ECM) is essential for muscle development and homeostasis [4]. The ECM is not static and undergoes 
remodeling throughout development and homeostasis, but it is not well understood how lysosomal-mediated protein turnover impacts muscle ECM composition and muscle structure. For example, the isoform of the heterotrimeric protein laminin changes during muscle development in amniotes and zebrafish [5,6]. Laminin 111 is the major constituent during initial muscle development. After initial muscle development laminin 111 is replaced with laminin 211. However, several lines of evidence suggest that there are reciprocal interactions between laminin and the autophagic/endocytic pathways. A laminin receptor, dystroglycan, plays a role in regulating the endocytosis and subsequent targeting to lysosomes for degradation of laminin 111 in mammary epithelial cells [7]. In addition, autophagy is differentially disrupted in two muscular dystrophies that result from mutations in extracellular matrix proteins. Whereas autophagy is increased in laminin $\alpha 2$ mutant mice and inhibition of autophagy rescues the phenotype; autophagy is decreased in collagen VI mutant mice and potentiation of autophagy rescues the phenotype $[8,9]$. Taken together, the above data clearly indicate that the autophagic/endocytic pathways and cell adhesion interact, but the consequences of altered lysosome function on the extracellular matrix during muscle development and homeostasis are not known.

Lysosomal $\mathrm{pH}$ is generated and maintained by lysosomal proton pumps. Optimal lysosomal $\mathrm{pH}$ is maintained by a vacuolar-type $\mathrm{H}^{+}$-ATPase (V-ATPase) that pumps protons into the lysosome, and multiple antiporters and symporters that move protons out of the lysosome [10-12]. V-ATPase is the primary driver of acidification within the endocytic pathway and the lysosome. Bafilomycin AI (BafA1), a specific inhibitor of V-ATPase, is able to block all lysosomal degradation that depends on the acidic $\mathrm{pH}$, thus illustrating the requirement for V-ATPase in lysosomal protein degradation. Sphingolipid transporter 1 (SPNS1), a highly conserved gene that encodes a putative late endosome/lysosome carbohydrate $/ \mathrm{H}^{+}$symporter, plays a pivotal role in maintaining optimal lysosomal pH [13]. Disruption of spns1 has been shown to cause excessive lysosomal accumulation and increased senescence in zebrafish embryos [12]. Here, we used the zebrafish mutant spns1 $1^{-/}$to investigate the requirement for normal lysosomal $\mathrm{pH}$ during muscle development and homeostasis.

In this study, we showed that dysregulated lysosomal $\mathrm{pH}$ disrupts muscle homeostasis and causes muscle degeneration. We found that the ECM protein laminin 111 was abnormally re-expressed at the myotendinous junction (MTF) in spns $1^{-/-}$muscle at 3.5 days post-fertilization (dpf). We hypothesize that laminin 111 expression is a compensatory response because double mutants for spns1 and a laminin receptor, Integrin $\alpha 7$ (Itga7), showed significantly worse muscle degeneration than either single mutant. However, the increased laminin 111 is not sufficient because there is still muscle degeneration in spns1 mutants. We hypothesized that lysosomal dysfunction decreased muscle membrane stability because this stability would likely not be fully rescued with the increased adhesion to the ECM with upregulated laminin 111. Experiments using Evans Blue Dye to identify membrane damage corroborated this hypothesis. Muscle degeneration in the zebrafish model of Duchenne muscular dystrophy (DMD) also predominantly results from sarcolemmal instability. Given the correlation between DMD-induced muscle damage due to sarcolemmal instability and increased levels of mmp9 in DMD [14], we investigated $m m p 9$ expression and detected upregulation of $m m p 9$ in $s p n s 1^{-1-}$ larvae. In contrast to the beneficial impact of inhibiting Mmp9 in mouse models of DMD [15], inhibition of mmp9 expression did not reduce muscle degeneration in $s p n s 1^{-1-}$ zebrafish. Together, these results suggest that dysregulated lysosomal $\mathrm{pH}$ correlates with altered expression of adhesion complex components at the MTJ, and some of these changes partially mitigate the muscle defects.

\section{Materials and Methods}

\subsection{Zebrafish Husbandry/Mutant/Transgenic Lines}

Zebrafish embryos were retrieved from natural spawns of adult zebrafish maintained on $14 \mathrm{~h}$ light $/ 10 \mathrm{~h}$ dark cycle. Spns $1^{\text {his91/h891 }}$ mutants were a generous gift from the Kishi 
lab at the Scripps Research Institute. Strains used in this study were spns $1^{\text {hi891/h891 [16], }}$

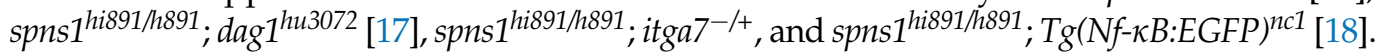
Embryos were grown in embryo rearing media $(1 \times \mathrm{ERM})$ with methylene blue at 28.5 degrees Celsius and staged according to [19].

\subsection{Itga7 Mutant Construction}

Itga7 crispr mutation was generated following a previously published protocol [20]. The single guide RNA (sgRNA) targeted DNA sequence of the Itga7 gene located in exon2, (NCBI Reference Sequence: XM_017358253.2), was selected by CHOPCHOP web tool (https: / / chopchop.cbu.uib.no, accessed on 15 April 2015). All sequences of the oligonucleotides used in this study are described in (Table 1). The single guide RNA oligo sequence (sgRNA) with protein were injected into a single cell stage, fertilized, wild type zebrafish embryos resulting in a 5 bp deletion (Scheme 1).

Table 1. Oligonucleotides used to generate Itga7 mutants.

\begin{tabular}{cc}
\hline Name & Sequence $\left(\mathbf{5}^{\prime} \rightarrow \mathbf{3}^{\prime}\right)$ \\
\hline Itga7 target site (PAM) & GGACCCTCACCTCTGGCCTG(AGG) \\
\hline $\begin{array}{c}\text { Itga7 specific oligonuclutide } \\
(\text { sp }+ \text { target-last } 3 \text { letters + overlap region) }\end{array}$ & ATTTAGGTGACACTATAGGACCCTCACCTCTGGCCTGGTTTTAGAGCTAGAAATAGCAAG \\
\hline $\begin{array}{c}\text { Itga7 stop-Cassette oligonuclutide } \\
\text { left homology + stop cassette + right homology) }\end{array}$ & TTCTGGTTGGAGCTCCTCAGGTCATGGCGTTTAAACCTTAATTAAGCTGTTGTAGGCCAGAGGTGAGGGTCCCCT \\
\hline Left Homology & TTCTGGTTGGAGCTCCTCAG \\
\hline Right Homology & GCCAGAGGTGAGGGTCCCCT \\
\hline
\end{tabular}

A

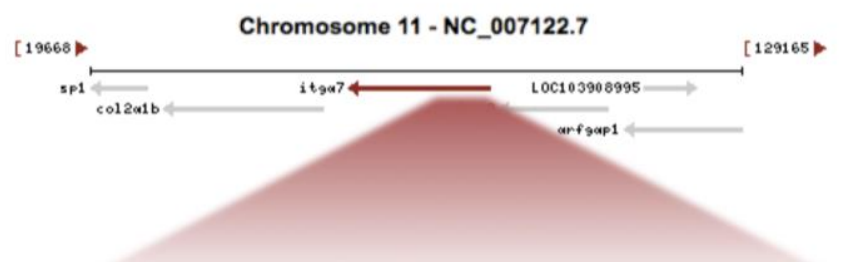

B

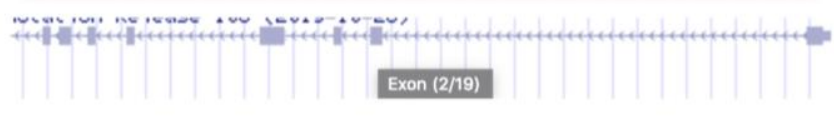

C

5' GCTCTGCAGGGGACCCTCACCTCTGGCCTGAGGAGCTCCAACC 3'

\section{C1} CCTCAGGCCAGAGGTGAGGGTCC

C1 CCTCAG- - - - -AGGTGAGGGTCC

Scheme 1. Schematic of the zebrafish Itga7 locus and CRISPR/Cas9 targeted region. (A) Itga7 location at chromosome 11. (B) Zoomed-in to itga7 exons. (C) Wild-type DNA sequence, the green is the CRISPR target, and the red is PAM site. (C1) Itga $7^{+/-}$sibling sequence. $\left(\mathbf{C} \mathbf{1}^{\prime}\right)$ Itga $7^{-/-}$mutant sequence with 5 bp deletion, allele designation itga $7^{\text {mai18 }}$.

\subsection{Morpholino Injections}

Morpholinos (MOs) were obtained from Gene Tools, LLC. MOs were diluted in sterile water to a stock concentration of $1 \mathrm{mM}$. Two itga6 translation-blocking MOs had the following sequences: MO1 5'-AGCTCCATTGCCTGAAATGAATG-3 ${ }^{\prime}$ and MO2 $5^{\prime}$-CTGTTGTATGAAAAATATAGCCCTT-3'. These two MOs were co-injected so that embryos received $9 \mathrm{ng} \mathrm{MO} 1$ and $8 \mathrm{ng} \mathrm{MO} 2$.

\subsection{Cyclopamine (CyA)/Aurintricarboxylic Acid (ATA) Treatment}

Embryos were treated with CyA to disrupt Sonic hedgehog (Shh) signaling. CyA dilution was chosen based on [21]. CyA (Toronto Chemical) was dissolved in 100\% EtOH to a stock concentration of $5 \mathrm{mM}$. $250 \mathrm{uL}$ of the $5 \mathrm{mM}$ stock was added to $25 \mathrm{~mL}$ of $1 \times$ ERM, 
bringing the final concentration to $50 \mathrm{uM} \mathrm{CyA}$ and $1 \% \mathrm{EtOH}$. Control embryos were treated with $1 \% \mathrm{EtOH}$.

Embryos were treated with ATA to disrupt TWEAK signaling. ATA dilution was chosen based on [22]. ATA (Sigma-Aldrich, St. Louis, MO, USA) was dissolved in 100\% EtOH to a stock concentration of $100 \mathrm{mM}$. $50 \mathrm{uL}$ of $100 \mathrm{mM}$ stock was added to $50 \mathrm{~mL}$ of $1 \times \mathrm{ERM}$, bringing the final concentration to $100 \mathrm{uM} \mathrm{ATA}$ and $0.1 \% \mathrm{EtOH}$. Control embryos were treated with $0.1 \% \mathrm{EtOH}$.

\subsection{Evans Blue Dye (EBD) Injection}

Zebrafish were anesthetized in $0.16 \mathrm{mg} / \mathrm{mL}$ tricaine in $1 \times$ ERM and side mounted on $1 \%$ agarose-lined Petri dishes in minimal volume of liquid. EBD $(1 \% w / v)$ was dissolved in a $0.9 \%$ saline solution. Injection needles were pulled from glass capillary tubes containing a filament on a Sutter Flaming/Brown Micropipette Puller. EBD solution was loaded into injection needles and injected into the circulation of anaesthetized $\sim 72 \mathrm{hpf}$ zebrafish using a MMPI-3 pressure injector from ASI. EBD injected zebrafish were maintained in $1 \times$ ERM for approximately $12 \mathrm{~h}$ before live imaging.

\subsection{Phalloidin Staining and Immunohistochemistry}

All antibodies (Abs) were diluted in block ( $5 \% w / v$ bovine serum albumin (BSA) in phosphate buffered saline (PBS) with $0.1 \%$ Tween20). Alexa 546 phalloidin (molecular probes) staining involved fixing embryos and larvae in $4 \%$ paraformaldehyde (PFA) for $4 \mathrm{~h}$ at room temperature (RT), washing 5 times for 5 min each $\left(5 \times 5^{\prime}\right)$ in $0.1 \%$ PBS-Tween, permeabilizing for $1.5 \mathrm{~h}$ in $2 \%$ PBS-Triton, washing $5 \times 5$ and then incubating in phalloidin (1:20) for $1-4 \mathrm{~h}$ at RT. Ab staining followed phalloidin staining. Ab staining involved blocking for $1 \mathrm{~h}$ at RT, incubating in $1^{\circ} \mathrm{Ab}$ overnight at $4{ }^{\circ} \mathrm{C}$, blocking for $8 \mathrm{~h}$ at RT, incubating in $2^{\circ} \mathrm{Ab}$ overnight at $4{ }^{\circ} \mathrm{C}$, then washing for $1 \mathrm{~h}$. $1^{\circ}$ Abs: anti-Laminin-111 1:50 (Sigma); anti-pY-397-FAK 1:50 (Biosource); anti-beta-dystroglycan 1:50 (Novocastra); anti-MMP-9 (GeneTEX). $2^{\circ}$ Abs: GAM/GAR 488, 546, 633 1:200 (Invitrogen).

\subsection{Comparative $q R T-P C R$}

RNA was isolated from whole embryos at $3.5 \mathrm{dpf}$ via Quiagen's RNeasy Mini Kit (Germantown, MD, USA). One-step comparative qRT-PCR was conducted with Quanta kit reagents and SYBR ${ }^{\circledR}$ Green. An Mx400 machine was used and the annealing temperature was at $60{ }^{\circ} \mathrm{C}$. Approximately $50 \mathrm{ng}$ of RNA template was used per reaction. We used betaactin as our normalizing transcript. See Table 1 for a complete list of primer information. See Table 2 for qRT-PCR results.

Table 2. Primer Sequences, Product Sizes and Concentrations.

\begin{tabular}{cccc}
\hline Primer & Sequence & Product Size & Final Conc. \\
\hline tweak forward & $5^{\prime}$-TGAATATGAGCAGGGCAGT-3 & $168 \mathrm{bp}$ & $100 \mathrm{uM}$ \\
\hline tweak reverse & $5^{\prime}$-TTCAATGCACTGGAGCAAAG-3' & & $100 \mathrm{uM}$ \\
\hline$m m p 9$ forward & $5^{\prime}$-TGATGTGCTTGGACCACGTAA-3' & $102 \mathrm{bp}$ & \\
\hline$m m p 9$ reverse & 5'-ACAGGAGCACCTTGCCTTTTC-3' & & \\
\hline
\end{tabular}

\subsection{Imaging}

Images were taken on an Olympus Fluoview IX-81 inverted microscope with FV1000 confocal system or a Leica TCS SP8 confocal system using the LASX Software. Embryos and larvae were side-mounted in $80 \%$ glycerol $/ 20 \%$ PBS and viewed with the $20 \times$ or $25 \times$ objective. Exposure times were kept constant throughout the imaging of an experiment allowing for the comparison of fluorescent levels between images. Live imaging for EBD experiments involved side mounting $\sim 3.5 \mathrm{dpf}$ zebrafish larvae in $0.4 \%$ low melt agarose containing $0.16 \mathrm{mg} / \mathrm{mL}$ tricaine and imaging via confocal microscopy. 


\subsection{Birefringence}

Birefringence was used to quantify the anisotropy of muscle structure [23]. Zebrafish were anesthetized with tricaine and transferred to glass bottom dishes prior to imaging. A Leica MZ10F stereomicroscope, attached DMC5400 camera, and polarizing filters were used to capture images. Imaging parameters, such as magnification, exposure, and gain were kept consistent throughout the experiment. Mean gray values were calculated from birefringent images in FIJI software by outlining fish three independent times and taking the mean of the gray values. The average mean gray value of each embryo was normalized by dividing by the average mean gray value of the ethanol treated sibling controls.

\subsection{Analysis and Statistics}

NF- $\kappa$ B activity was quantified using mean grey values of fluorescent intensity based on [24]. Fluorescent images were captured at $25 \times$ magnification. Fluorescence signal intensity was determined using mean grey values with images converted to a pre-set grey scale (ImageJ). All image capturing and threshold parameters were kept constant between experiments.

Categorical data were analyzed using $\mathrm{R}$ version 3.5.1 using Fisher's exact test. Immunohistochemistry data was visually scored. Relative fluorescence intensity was assigned a score of 0 (no staining), 1 (weak staining), or 2 (strong staining).

Quantitative data were analyzed using Prism7. Quantitative statistical comparisons of two groups were conducted using student $t$-tests. Quantitative statistical comparisons of multiple groups were conducted using ANOVA followed by Tukey's honest significant difference multiple comparisons test.

\section{Results}

3.1. Initial Muscle Development Proceeded Normally But Degeneration Began at 3.5 Days Post-Fertilization

As has been previously shown, spns $1^{-/-}$mutant larvae show increased lysosomes by $3.5 \mathrm{dpf}$ (Figure 1A,B) $[12,25,26]$. Although beta-galactosidase [16] has been observed in spns $1^{-/-}$muscle, the effects of lacking spns1 on muscle structure have not previously been reported. In addition, it is not known whether muscle development proceeds normally in spns1 $1^{-/-}$embryos prior to beta-galactosidase expression. We found that initial muscle development proceeded normally, where myotomes in $s p n s 1^{-/-}$mutants were similar to wild-type embryos at $1 \mathrm{dpf}$ (Figure 1C,D). By $3.5 \mathrm{dpf}$, spns1-/- mutants began to display slightly unorganized fibers and myotendinous junctions (MTJs) (Figure 1E,F). Overt degeneration of muscle fibers also began at $3.5 \mathrm{dpf}$ (Figure 1H). Fiber-type (slow-twitch or fast-twitch) susceptibility to muscle damage varies between different muscle-related disorders [27]. Interestingly, both fast-twitch and slow-twitch fibers were damaged in spns $1^{-1-}$ larvae. There were no significant differences in the proportion of $s p n s 1^{-1-}$ embryos that displayed fast-twitch fiber detachments vs. slow-twitch fiber detachments $(p$-value $=0.8018)$ (Figure 1I). The severity of muscle degeneration was also similar: there were no significant differences in the proportion of segments per embryo that displayed fast-twitch fiber detachments vs. slow-twitch fiber detachments $(p$-value $=0.5748)($ Figure $1 \mathrm{~J})$. This data indicated that both fast-twitch and slow-twitch muscles were damaged in spns1 ${ }^{-1-}$ larvae. However, muscle damage in individual spns $1^{-/-}$larvae was usually restricted to one fiber type. The proportion of spns $1^{-/-}$larvae that contained only fast-twitch detachments or only slow-twitch detachments was significantly greater than $s p n s 1^{-/-}$larvae that contained both fast-twitch and slow-twitch muscle fiber detachments ( $n=27 \mathrm{spns} 1^{-1-}$ larvae, $p$-value $=0.0023, p$-value $=0.0003$, respectively) (Figure 1I). Thus, although both fiber types were susceptible to damage, fiber detachments in individual spns $1^{-/-}$larvae had a tendency to be restricted to a single fiber type. The mechanism mediating this effect is unknown. Taken together, these data suggest that dysregulated lysosomal $\mathrm{pH}$ did not impact initial muscle development, but disrupted muscle and MTJ homeostasis. 

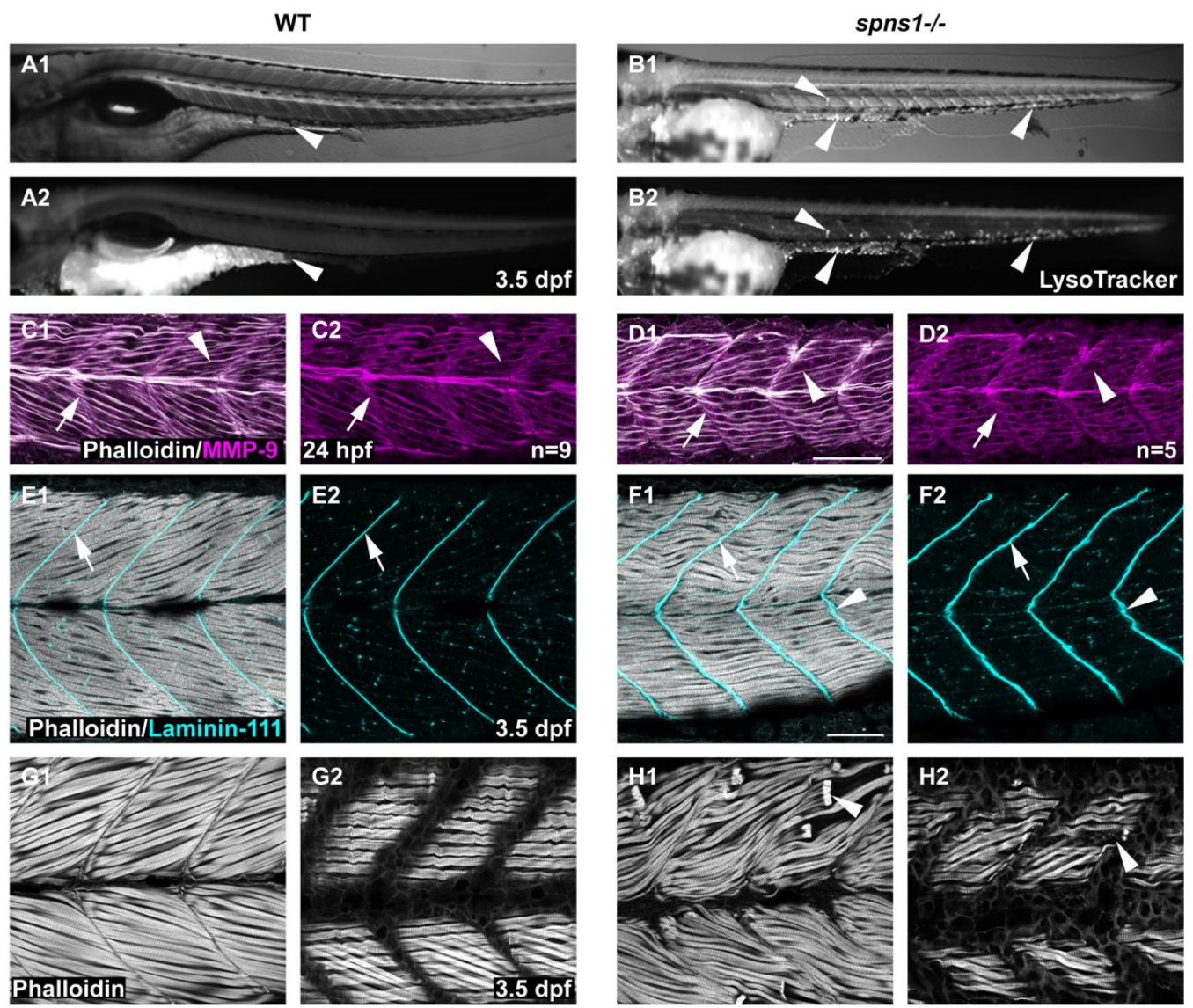

I: \% spns $1^{-1}$ embryos with muscle degeneration
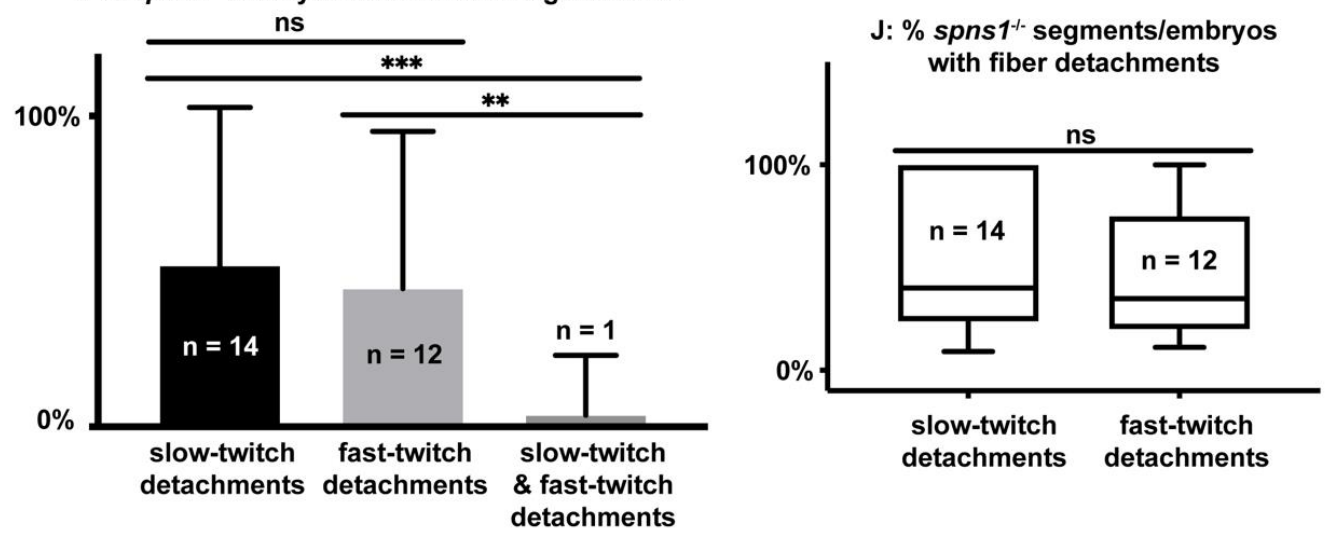

Figure 1. Developmental characterization of spns1 $1^{-/}$embryos and larvae. Anterior left, dorsal top, side-mounted embryos and larvae. (A1,B1) WT and (A2,B2) spns1 ${ }^{-/-}$brightfield and LysoTracker staining at 3.5 dpf. Arrowheads indicate enlarged LysoTracker positive puncta in spns1 $1^{-/}$larvae. (C1-D2) Phalloidin staining (white) for actin and MMP-9 antibody (purple) staining in $1 \mathrm{dpf}$ embryos. (C1) Merged WT and (D1) spns1 $1^{-1-}$ channels and (C2,D2) single MMP-9 channel. Arrows indicate MMP-9 staining at the MTJ while arrowheads indicate MMP-9 staining localized at muscle fibers. Scale bar $50 \mu \mathrm{m}$ (E1-F2) Phalloidin staining (white) and beta-dystroglycan antibody (cyan) staining in 3.5 dpf larvae. (E1) WT and (F1) spns1 ${ }^{-/-}$merged channels and (E2,F2) beta-dystroglycan single channel. Arrows indicate beta-dystroglycan staining at the MTJ while arrowheads indicate wavy beta-dystroglycan staining at the MTJ in spns1 ${ }^{-/-}$larvae. Scale bar $50 \mu \mathrm{m}$. $(\mathrm{G} 1, \mathrm{G} 2, \mathrm{H1}, \mathrm{H} 2)$ Phalloidin staining of WT and spns1-/- fast and slow muscle fibers in $3.5 \mathrm{dpf}$ larvae. Arrowheads indicate fiber detachments in spns1 ${ }^{-/-}$larvae. Scale same as (F1). (I) Quantification of $s p n s 1^{-/}$larvae $(n=27)$ with slow, fast, or both types of muscle fiber detachments. (J) Quantification of the percent of total myotomes in spns1 $1^{-/-}$larvae $(n=27)$ with fast or slow muscle fiber detachments. Box and Whisker plot with whiskers denoting minimum and maximum values. ${ }^{* *}$ for $p<0.01$, and ${ }^{* * *}$ for $p<0.001$. 
3.2. Aberrant Upregulation of the Extracellular Matrix Protein Laminin 111 at the MTJ Coincided with the Onset of Muscle Degeneration

The muscle extracellular matrix is critical for muscle development and homeostasis [28]. Although initial muscle development proceeded normally in spns1 $1^{-/}$embryos, we observed disrupted muscle and MTJ structure at $3.5 \mathrm{dpf}$. We next asked when abnormal muscle/MTJ structure first appears in spns1 ${ }^{-/}$mutants. In wild-type embryos, skeletal muscle fibers grow as development proceeds (note thicker fibers in panel C1 relative to panel B1). This initial growth also occurred in spns1 $1^{-/-}$mutants (compare G1 to F1). At $3 \mathrm{dpf}$, skeletal muscle fibers in spns1-/- mutants appeared quite similar to wild-type fibers. However, just several hours later fibers in $s p n s 1^{-/-}$embryos were disorganized and began to degenerate (Figure 1(H1,H2), white arrowhead).

The MTJ also developed normally but was disrupted at $3.5 \mathrm{dpf}$. The basement membrane protein laminin 111 was highly concentrated at the MTJ during the first couple of days of development in both wild-type and spns1 ${ }^{-1-}$ mutants (Figure 2A,B,E,F). In wild-type embryos, the developmental laminin 111 isoform was then replaced by laminin 211 such that by $3.5 \mathrm{dpf}$ laminin 111 is absent from wild-type MTJs (Figure 2C). This pattern was also observed in spns $1^{-1-}$ mutants (Figure 2D). However, by $3.5 \mathrm{dpf}$ robust expression of laminin 111 reappeared at the MTJ in spns $1^{-/-}$mutants (Figure 2F). We quantified laminin 111 staining by assigning a qualitative score of laminin 111 staining to blinded images. Relative laminin 111 staining at the MTJ in WT controls was significantly weaker at $3.5 \mathrm{dpf}$ compared to 1 and $2 \mathrm{dpf}$ ( $p$-values < 0.0001) (Figure 2(C2) vs. Figure 2(A2,B2)). In spns $1^{-/}$mutants the relative laminin 111 staining at the MTJ was significantly weaker at $3 \mathrm{dpf}$ compared to $1 \mathrm{dpf}(p$-value $=0.006)$ (Figure 2(G2) vs. Figure 2(E2)). In fact, there was no significant difference in the relative laminin 111 staining at the MTJ in spns1 $1^{-/-}$ mutants at $3.5 \mathrm{dpf}$ vs. $1 \mathrm{dpf}$ ( $p$-value $>0.9999$ ) (Figure 2J). These data indicate that laminin 111 was re-expressed in spns $1^{-/-}$muscle at a time when it is not normally expressed in WT controls.

\subsection{A Different Mechanism Underlies Laminin 111 Re-Expression Than Underlies Laminin 111 Expression during Initial MTJ Development}

Shh signaling is required for normal laminin 111 expression at the developing MTJ $[29,30]$. As laminin 111 was re-expressed after downregulation in spns1 $1^{-/}$muscle, we hypothesized that Shh signaling would also be required for laminin 111 re-expression in spns $1^{-/-}$ larvae. Cyclopamine blocks Shh signaling [31]. Cyclopamine was administered beginning at $2 \mathrm{dpf}$ to avoid disruption of initial muscle development [32]. WT and spns $1^{-1-}$ embryos were treated with $50 \mu \mathrm{M}$ cyclopamine (with $1 \% \mathrm{EtoH}$ ) beginning at $2 \mathrm{dpf}$. Controls were treated with $1 \% \mathrm{EtOH}$. In wild-type control embryos treated with EtOH, laminin 111 was not expressed in the MTJ at $3.5 \mathrm{dpf}$ (Figure 3A). Surprisingly, laminin 111 was strongly concentrated at the MTJ in cyclopamine-treated wild-type embryos (Figure 3B). The relative laminin 111 concentrated at the MTJ in WT larvae treated with cyclopamine was significantly stronger than the laminin 111 staining in the WT controls ( $p$-value $<0.0001)$. In contrast, cyclopamine treatment had no effect on laminin 111 at the MTJ in spns $1^{-/-}$ larvae: there was no significant difference in the relative laminin 111 staining at the MTJ in spns $1^{-/-}$larvae treated with cyclopamine vs. control spns $1^{-1-}$ larvae $(p$-value $=0.1838)$ (Figure 3C,D). These data indicate that, in contrast to initial MTJ development, Shh was not required for laminin 111 re-expression in $s p n s 1^{-/-}$larvae. 


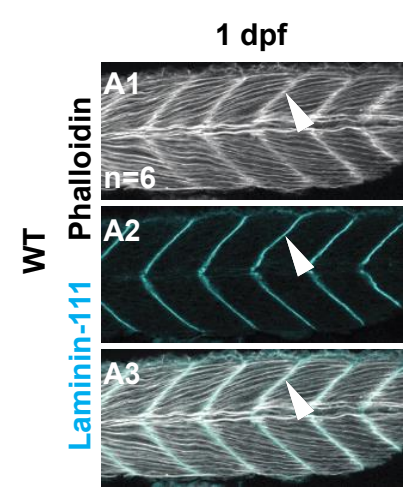

$1 \mathrm{dpf}$

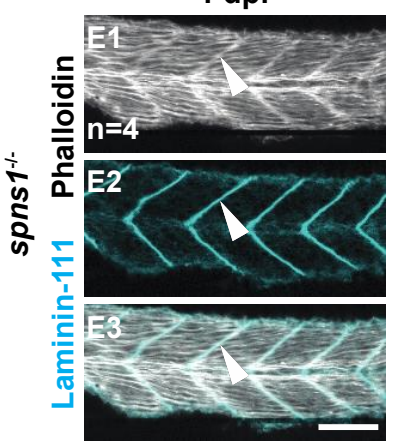

I. Relative anti-laminin-111 staining intensity at the MTJ

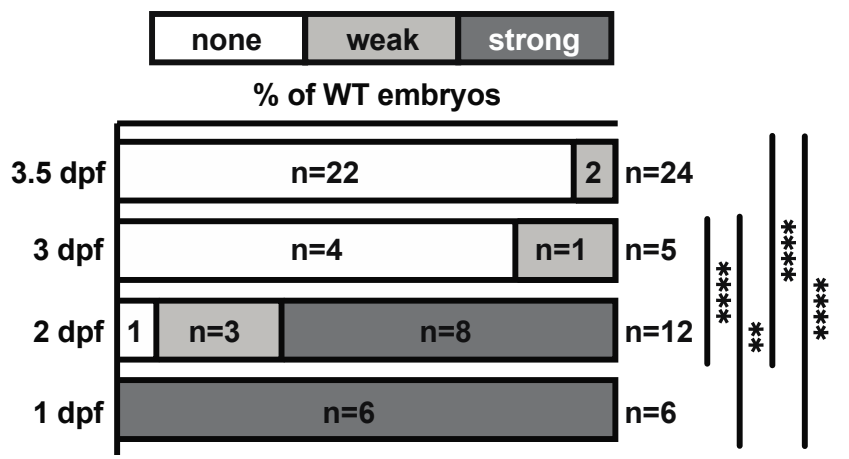

$3 \mathrm{dpf}$
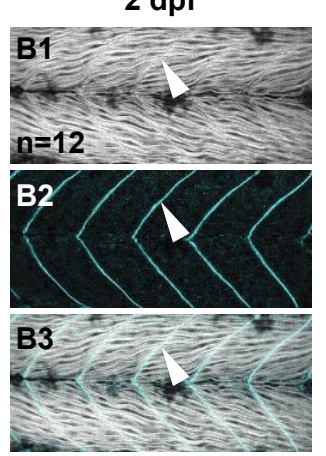

$2 \mathrm{dpf}$

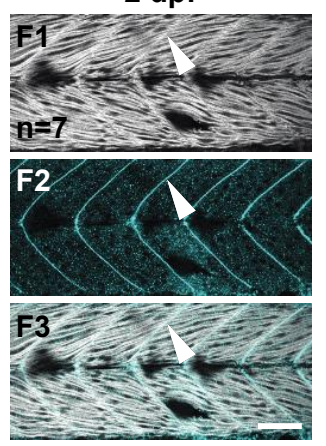

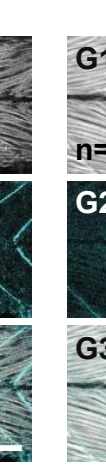
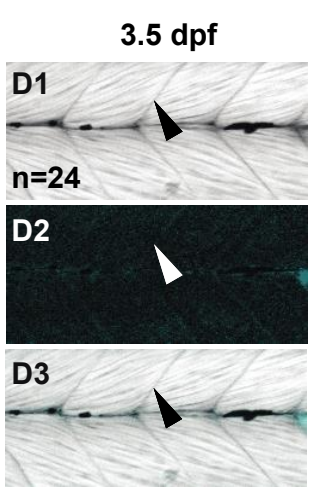

$3.5 \mathrm{dpf}$

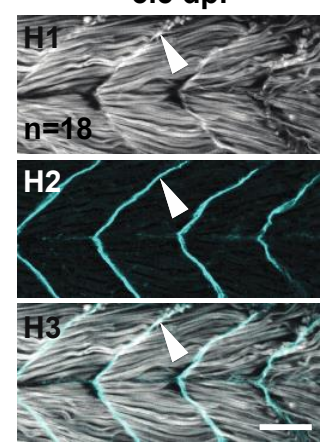

J. Relative anti-laminin-111 staining intensity at the MTJ

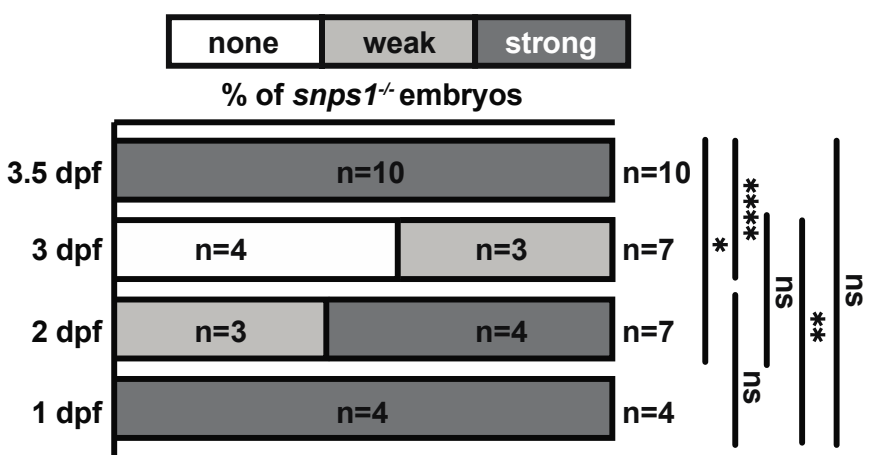

Figure 2. Normal initial muscle development is followed by abnormal $3.5 \mathrm{dpf}$ laminin-111 levels in spns1 ${ }^{-/-}$embryos and larvae. (A1-H3) Anterior left, dorsal top, side-mounted embryos and larvae stained with phalloidin (white) to visualize actin and laminin-111 antibodies (cyan). (A1-H1) Phalloidin staining. (A2-H2) Laminin-111 staining. (A3-H3) Merged phalloidin and laminin-111 channels. White arrowheads point to laminin-111 localized to the MTJ. Laminin-111 staining, not detected in WT embryos and larvae at 3-3.5 dpf, is detected in (H2-H3) spns1 ${ }^{-/}-3.5 \mathrm{dpf}$ larvae. (I,J) Relative fluorescent intensity of laminin-111 protein in WT and spns1 $1^{-/-}$embryos and larvae over time (see methods). ${ }^{*} p<0.05,{ }^{* *} p<0.01$, $* * * * p<0.0001$. Scale bars $50 \mu \mathrm{m}$. 

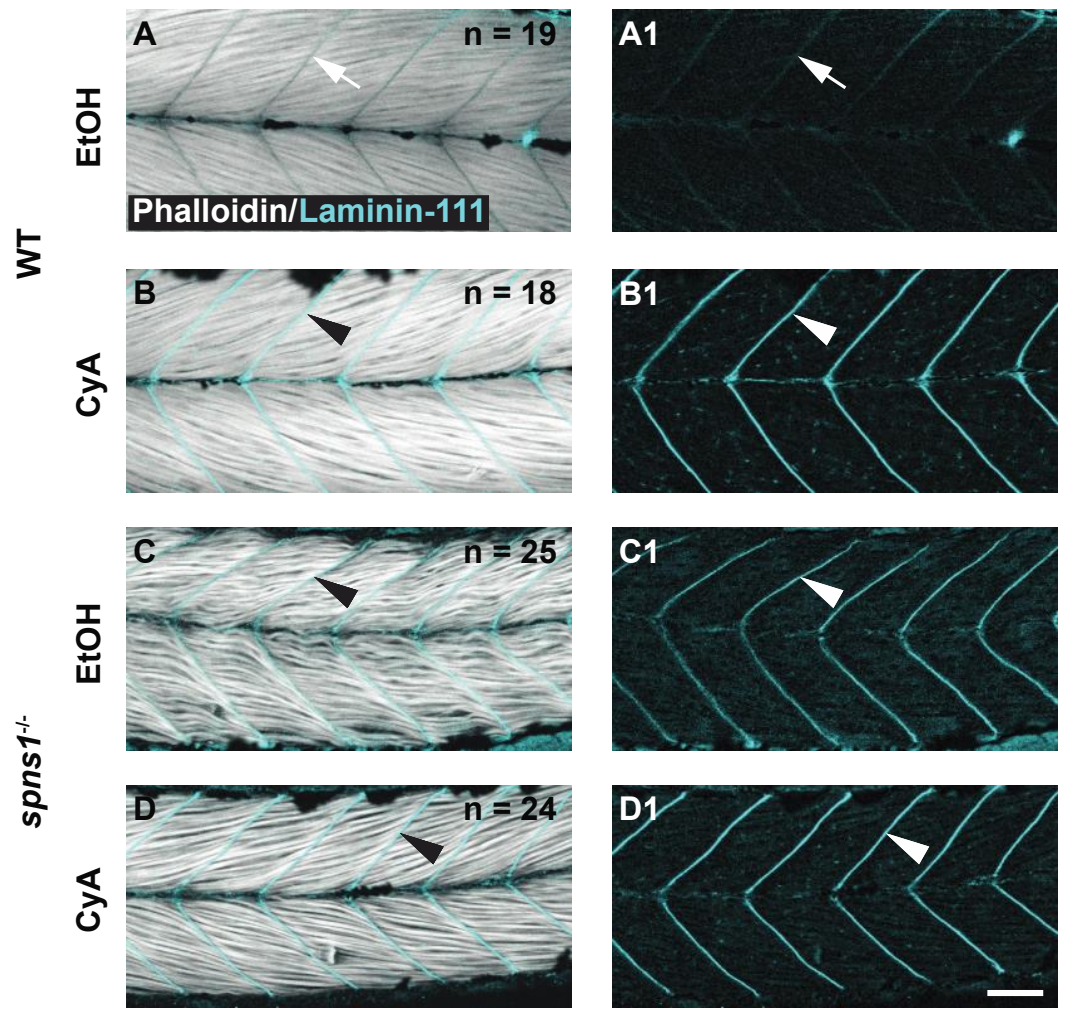

E: Relative anti-laminin-111 staining intensity at the MTJ

F: Relative anti-laminin-111 staining intensity at the MTJ
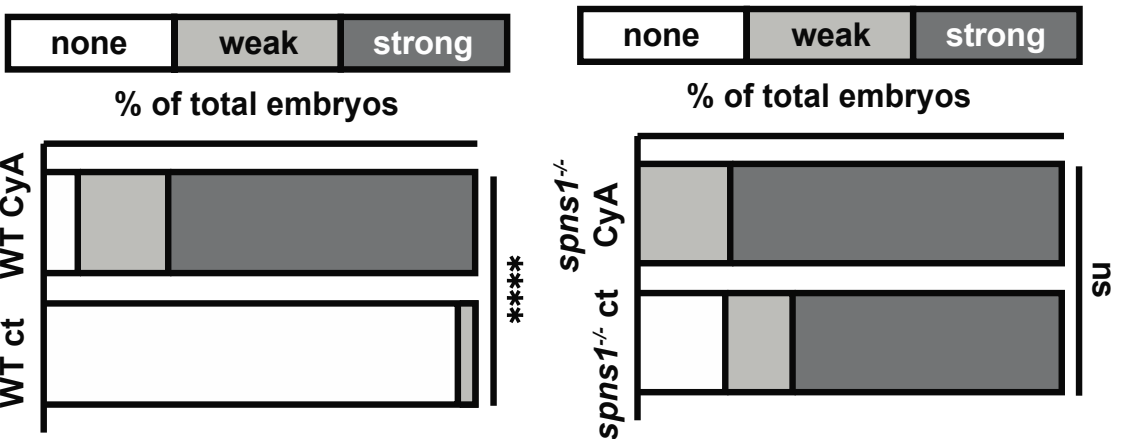

Figure 3. Sonic Hedgehog signaling is not required for laminin- 111 protein re-expression in $3.5 \mathrm{dpf}$ spns1 ${ }^{-/}$larvae. (A-D1) Anterior left, dorsal top, side-mounted $3.5 \mathrm{dpf}$ WT and spns1 ${ }^{-/-}$larvae stained with phalloidin (white) and laminin-111 (cyan) post vehicle control or post $50 \mu \mathrm{M}$ cyclopamine treatment. (A,A1,C,C1) Phalloidin and laminin-111 staining after EtOH vehicle control treatment in (A,A1) WT and (C,C1) spns1 $1^{-/-}$larvae. (A-A1) White arrows point to MTJ in WT larvae. Note absence of laminin-111 protein in WT embryos and presence of laminin-111 protein in spns1-/- larvae. (B,B1,D,D1) Phalloidin and laminin-111 staining after cyclopamine treatment in WT and spns1-/larvae. White arrowheads point to laminin-111 staining at the MTJ. (E,F) Relative fluorescent intensity of laminin-111 staining in (E) WT and (F) spns1 $1^{-/}$larvae after ethanol or cyclopamine exposure. **** $p<0.0001$. Scale bar $50 \mu \mathrm{m}$.

\subsection{The Laminin Receptor Dystroglycan Did Not Genetically Interact with Spinster}

The deposition and polymerization of laminin in the basement membrane is regulated by transmembrane cell-surface receptors [33], raising the possibility that these transmembrane receptors could be required for ectopic laminin 111 expression at $3.5 \mathrm{dpf}$ in spns $1^{-/-}$ mutants. In addition, mutations in laminin 211 or either of the two main transmembrane receptors that bind to laminin 211, dystroglycan (dag1) and integrin alpha 7 (itga7), result 
in muscular dystrophy [34,35]. Although laminin 111 is the developmental isoform, exogenous laminin 111 can decrease muscle degeneration when adhesion to laminin 211 is disrupted [35,36]. We hypothesized that: (1) re-expression of laminin 111 could be a compensatory mechanism to combat muscle degeneration that occurs in spns $1^{-/-}$mutants, and (2) either dag1 or itga7 could be required for ectopic laminin 111 expression in spns $1^{-/-}$ mutants. We first tested these hypotheses by generating dag1; spns1 double mutants. Expression of laminin 111 in dag1 mutants was similar to wild-type embryos: laminin 111 was robustly concentrated at the MTJ during initial muscle development (Figure 4A) and downregulated by $3 \mathrm{dpf}$ (Figure 4B). This downregulation was maintained at $3.5 \mathrm{dpf}$ (Figure 4C). In contrast, although laminin 111 expression was absent at $3 \mathrm{dpf}$ in dag1; spns1 ${ }^{-/-}$double mutants (Figure 4E), laminin 111 was re-expressed by $3.5 \mathrm{dpf}$ (Figure 4(F1)). This result indicated that dystroglycan was not necessary for re-expression of laminin 111 in spns $1^{-/-}$ mutants. In addition, the spns $1^{-/-}$phenotype was not exacerbated by lack of dystroglycan. Together, these data suggest that dystroglycan did not genetically interact with Spinster.
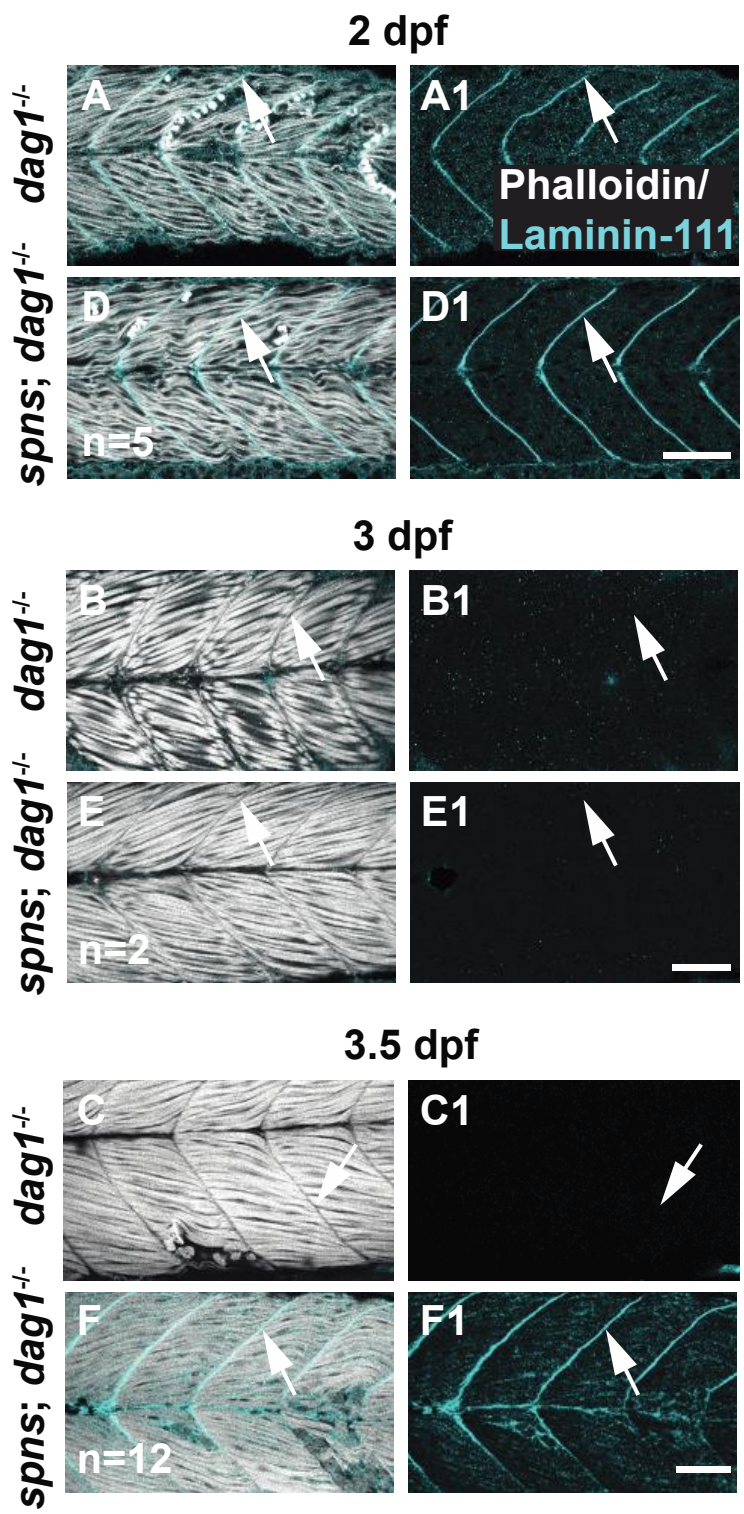

Figure 4. Cont. 


\section{G: Relative anti-laminin-111 staining intensity at the MTJ

\begin{tabular}{|l|l|l|}
\hline none & weak & strong \\
\hline
\end{tabular}

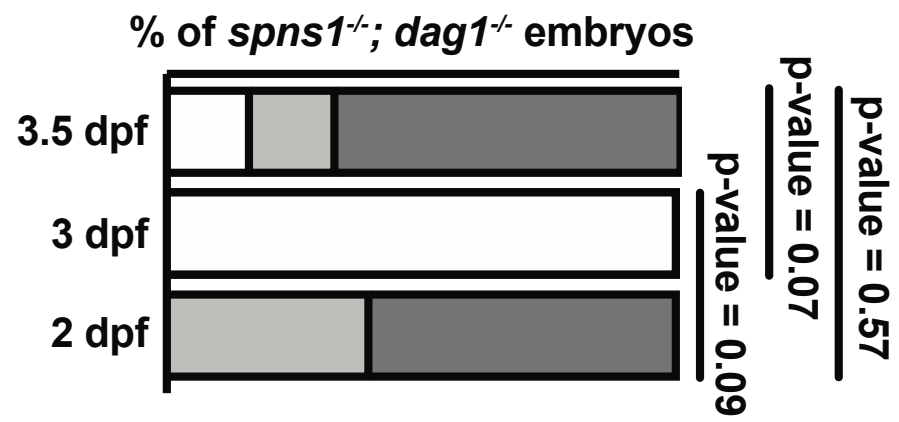

Figure 4. Laminin receptor beta-dystroglycan is not required for laminin-111 protein re-expression at $3.5 \mathrm{dpf}$ in spns1 ${ }^{-/-}$larvae. (A-F1) Anterior left, dorsal top, side-mounted embryos, and larvae. dag1 $1^{-/-}$and spns1 $1^{-/-}$; dag $1^{-/-}$embryos and larvae stained with phalloidin (white) and laminin111(cyan), (A-F) Merged phalloidin and laminin-111 channels and (A1-F1) single beta-dystroglycan channel over time. (A-F) White arrowheads point to dystrophic lesions and (A1-F1) white arrowheads point to laminin-111 staining. (G) Relative laminin-111 fluorescent intensity over time in spns1 $1^{-/} ;$dag1 $1^{-/-}$embryos and larvae. Scale bars $50 \mu \mathrm{m}$.

\subsection{The Laminin Receptor Integrin $\alpha 7$ Contributed to Muscle Homeostasis in spns1 $1^{-1-}$ Mutants}

We next asked whether itga7 was required for muscle homeostasis and laminin 111 re-expression in spns $1^{-/-}$larvae by generating itga7; spns1 double mutants. Laminin 111 was not expressed in itga7 mutants at $3.5 \mathrm{dpf}$ (Figure 5(B1)). In itga7; spns1 double mutants, laminin 111 was concentrated at the MTJ at 1 and $2 \mathrm{dpf}$ and downregulated by $3 \mathrm{dpf}$ (not shown). However, by $3.5 \mathrm{dpf}$ laminin 111 was re-expressed and concentrated at the MTJ in itga7;spns1 double mutants (Figure 5(D1), white arrow). Thus, itga7 was not required for laminin 111 re-expression in spns1 mutants. Mutations in Itga7 result in Itga7-linked congenital muscular dystrophy [34]. Muscle degeneration occurs in zebrafish deficient for itga7 [37], and in our itga7 mutant (Figure 5B, red arrowheads). We next tested whether lack of itga7 exacerbated muscle degeneration in spns $1^{-/}$mutants. Muscle degeneration was more severe in itga7; spns1 double mutant larvae (Figure 5D, yellow star). Significantly more segments per embryo exhibited degeneration in itga7; spns1 double mutant larvae compared to either single mutant (Figure 5E). Degenerated muscle fibers in itga7; spns1 double mutants are surrounded by laminin 111. This result suggests that itga7 was necessary for laminin 111 integrity at the MTJ in spns1 mutants. Together, these data suggest that Itg $\alpha 7$ contributed to muscle homeostasis in spns1 larvae.
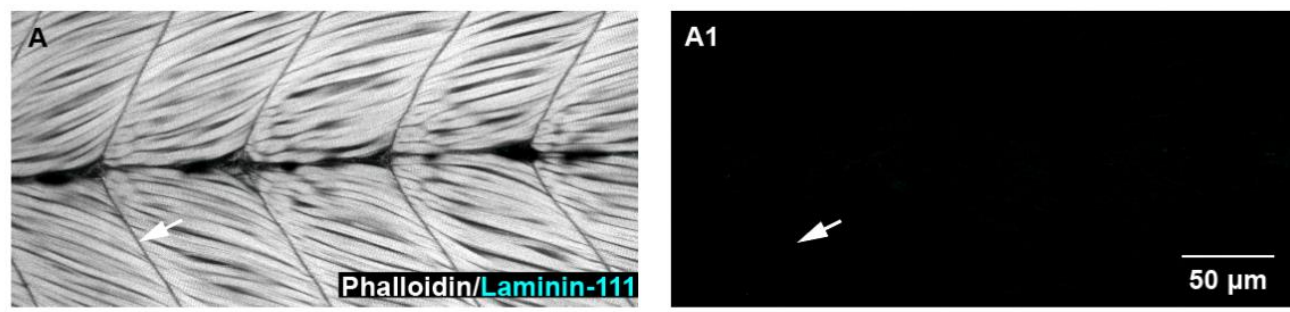

Figure 5. Cont. 

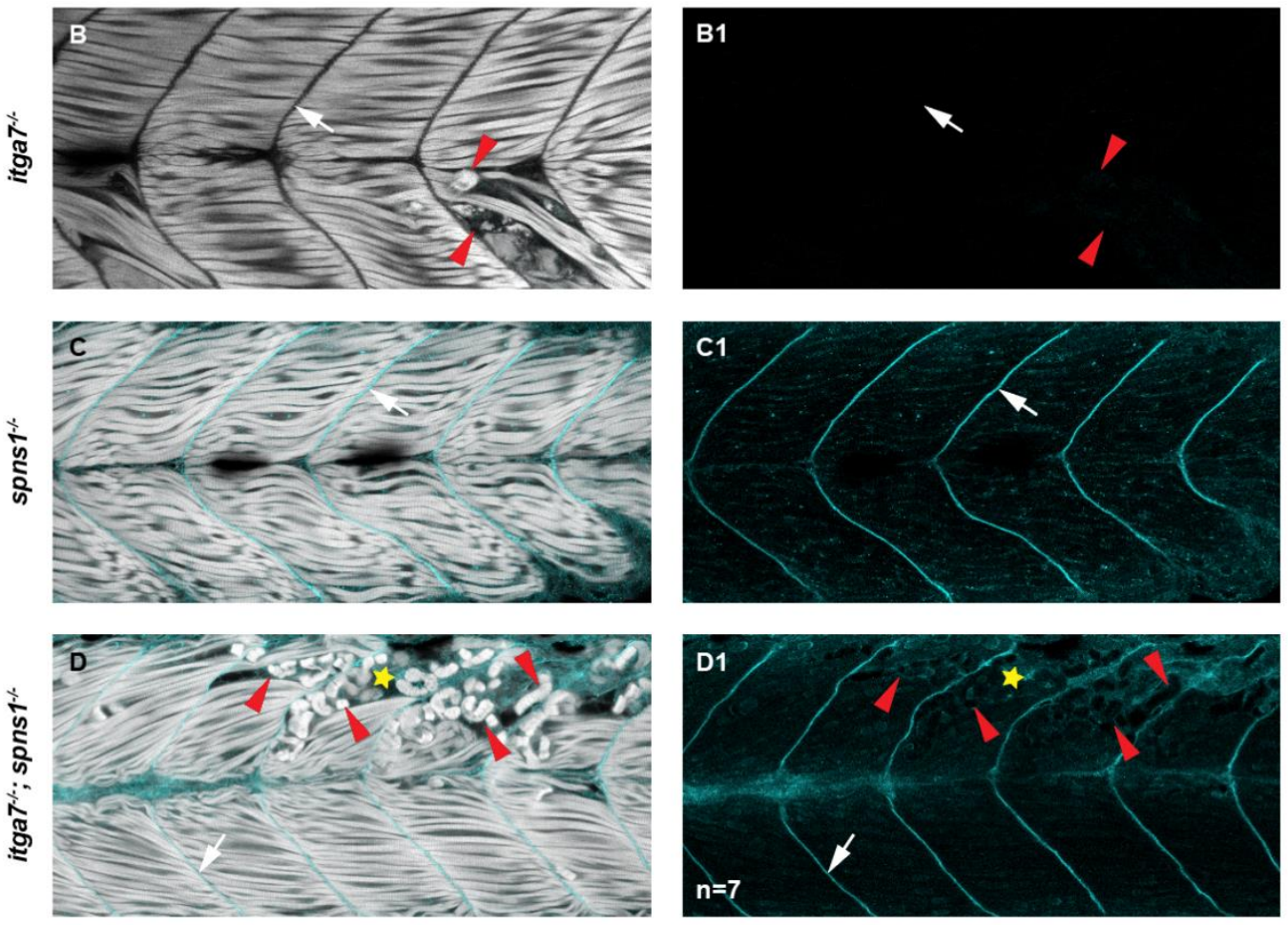

E: \% of myotomes/embryo with degeneration

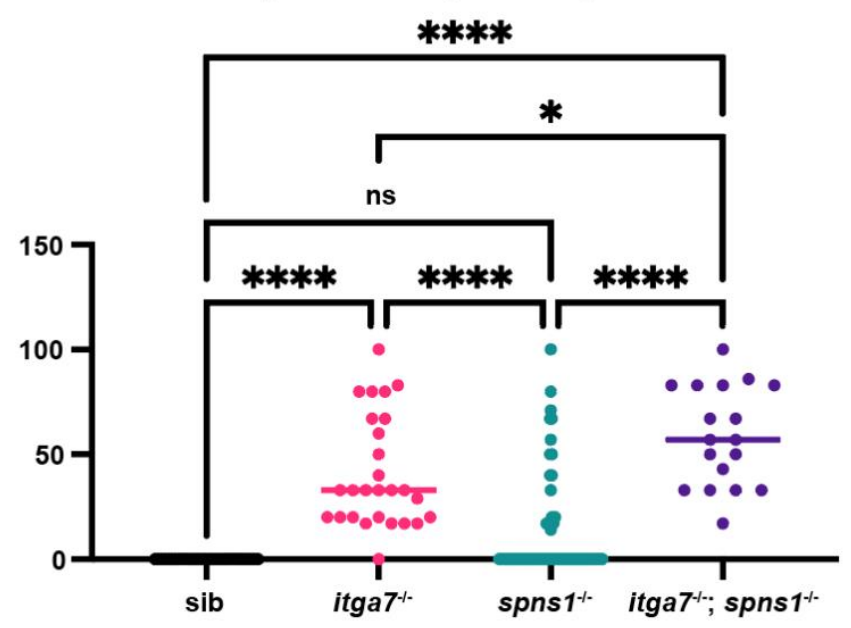

Figure 5. The laminin receptor integrin $\alpha 7$ is not required for laminin-111 protein re-expression at $3.5 \mathrm{dpf}$ in spns1-/larvae but does contribute to muscle resilience in $s p n s 1^{-/}$larvae. (A-D) Anterior left, dorsal top, side-mounted larvae. Phalloidin (white) and laminin-111 (cyan) stained WT, itga ${ }^{-/-}$, spns1 $1^{-/}$, and spns1 ${ }^{-/-}$; itga $7^{-/-}$larvae at $3.5 \mathrm{dpf}$. (A-D) Merged phalloidin and laminin-111 channels and (A1-D1) single laminin-111 channel. White arrows indicate the MTJ. Red arrowheads point to dystrophic lesions. Yellow star indicates aberrant laminin-111 surrounding detached fibers. (E) Percent of myotomes with dystrophic lesions. Note the significant increase in muscle degeneration in $\operatorname{spns} 1^{-1-} ;$ itga $7^{-/-}$larvae compared to itga $^{-/-}$single mutants. ${ }^{*} p<0.05,{ }^{* * * *} p<0.0001$. Scale bar $50 \mu \mathrm{m}$.

\subsection{Sarcolemmal Instability in spns1 Muscle Fibers}

The above data suggest the hypothesis that adhesion to laminin 111 was upregulated in a compensatory manner in spns1 mutants. Muscle damage in zebrafish models studied thus far can result from two distinct cellular etiologies: failure to maintain sarcolemmal integrity or failure to maintain adhesion at the MTJ external to the sarcolemma [6]. Muscle fibers degenerate in spns1 mutants, suggesting that adhesion to laminin 111 is not sufficient to promote muscle homeostasis in spns1 mutants. We thus hypothesized that muscle degeneration in spns $1^{-/-}$larvae was due to sarcolemmal instability. We used cell imper- 
meable Evans blue dye (EBD), which only fills muscle fibers when sarcolemmal integrity is compromised, and beta-dystroglycan (DG) antibody staining to distinguish between these two etiologies. EBD was observed in long, attached fibers in spns $1^{-/}$mutants at $3.5 \mathrm{dpf}$ (Figure 6B,C). This result indicated that sarcolemmal damage occurred prior to overt muscle degeneration. Detection of the membrane-integral protein beta-DG at the detached ends of retracted fibers suggested adhesion failure at the MTJ, while beta-DG retained at the MTJ indicated failure to maintain sarcolemmal integrity [38]. The proportion of spns1 $1^{-/}$mutants with fiber detachments showing the retention of beta-DG at the MTJ (Figure 6F) was significantly greater than the proportion of spns1 ${ }^{-1-}$ mutants showing the retention of beta-DG at the detached ends of retracted fibers $(p$-value $=0.0002)$ (Figure 6G) Additionally, the proportion of segments per spns $1^{-/}$mutant with fiber detachments showing retention of beta-DG at the MTJ was significantly greater than the proportion of segments showing the retention of beta-DG at the detached ends of retracted fibers ( $p$-value $<0.0001)$ (Figure 6D). These data indicate that fiber detachments in spns1 ${ }^{-1-}$ mutants predominantly resulted from sarcolemmal instability.

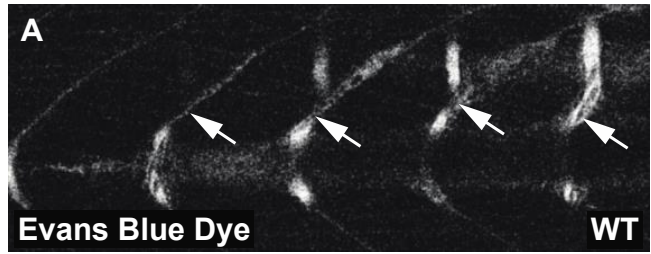

C: Fully elongated spns $1^{\text {-1- }}$ fibers take up EBD

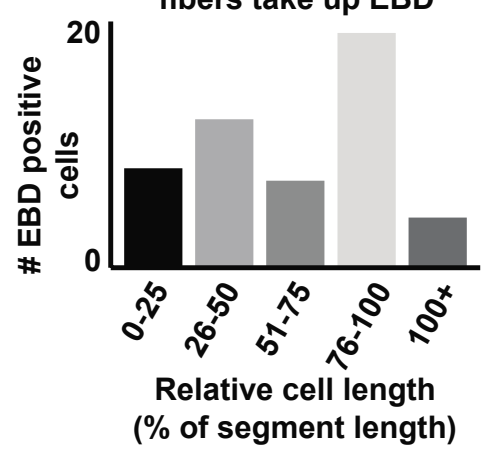

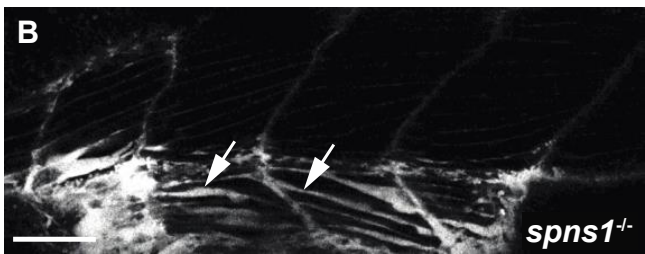

D: \% spns $1^{-/-}$segments with fiber detachments

E: \% spns $1^{-/}$embryos with fiber detachments
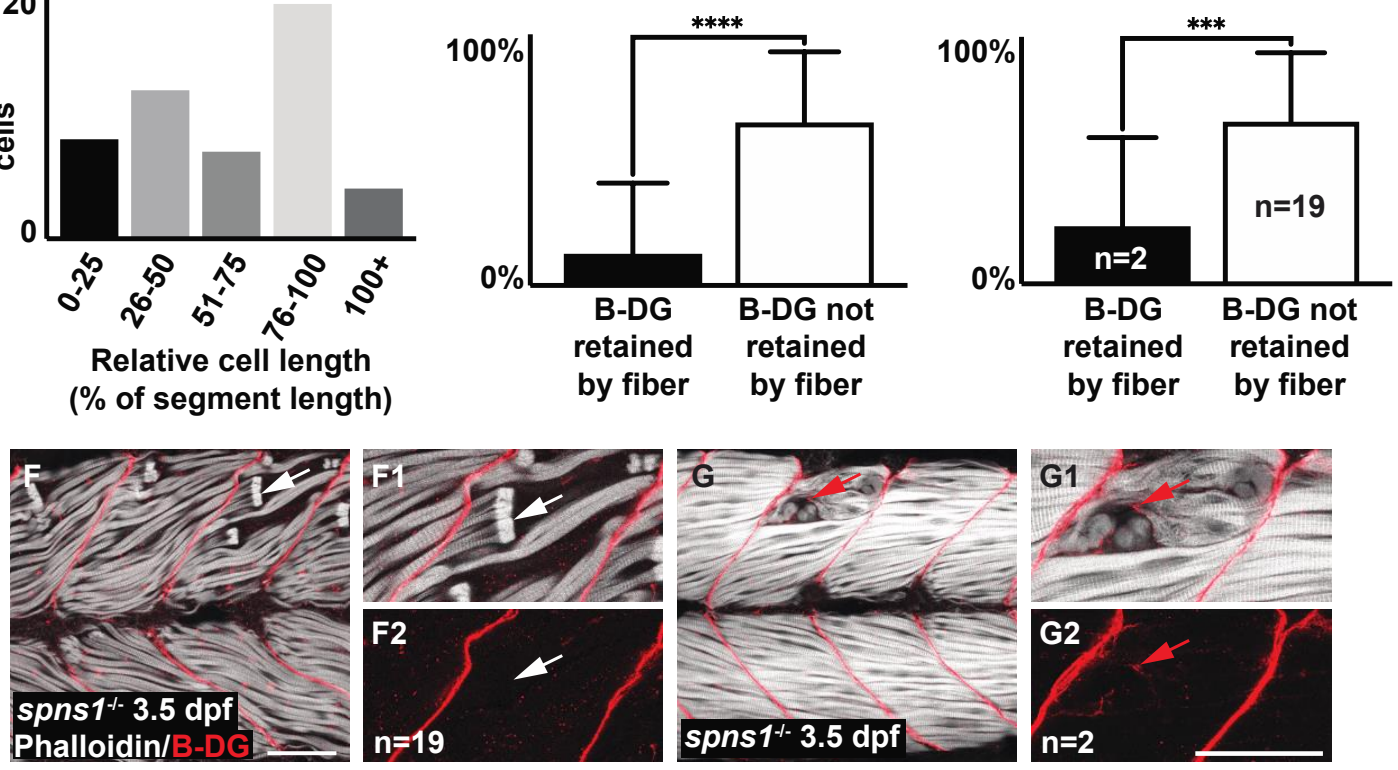

Figure 6. Muscle fiber failure is due to sarcolemmal integrity failure in $s p n s 1^{-/} 3.5$ dpf larvae. (A,B,F,G) Anterior left, dorsal top, side-mounted $3.5 \mathrm{dpf}$ larvae. (A,B) WT and spns1-/- $3.5 \mathrm{dpf}$ larvae injected with $1 \%$ Evans Blue Dye (EBD). (A,B) White arrows indicate the presence of EBD. (A) In WT larvae EBD remains in the bloodstream but in (B) spns1 ${ }^{-/-}$larvae EBD penetrates full length muscle fibers, indicating membrane damage. (C) Quantification of relative cell length of EBD-penetrated myofibers in spns1 $1^{-/}$larvae shows that fully elongated fibers in spns1 $1^{-/}$larva take up EBD. (D) Percentage of segments with beta-dystroglycan containing fibers and beta-dystroglycan negative fibers in spns1-/larvae. Note that beta-dystroglycan remains at the MTJ the majority of the time. (E) Percentage of spns1 $1^{-/-}$larvae with betadystroglycan containing fibers and beta-dystroglycan negative fibers. ${ }^{* * *} p<0.001{ }^{* * * *} p<0.0001$. (F-G2) Phalloidin (white) and beta-dystroglycan staining (red) of $3.5 \mathrm{dpf} s p n s 1^{-/-}$larvae. (F,F1,G,G1) Merged phalloidin and beta-dystroglycan channels and (F2,G2) beta-dystroglycan single channel. (F-F2) White arrows point to retracted fibers that do not contain beta-dystroglycan at their detached ends. (G-G2) Red arrows point to retracted fibers that contain beta-dystroglycan at their detached ends. Scale bars $50 \mu \mathrm{m}$. 


\subsection{MMP-9 Was Upregulated in spns $1^{-/-}$Mutants}

The above data indicate that while Itga7-mediated adhesion to laminin 111 contributed to muscle homeostasis in spns $1^{-/}$mutants, it was not sufficient because the instability of muscle membranes in spns1 $1^{-1-}$ mutants also contributed to muscle degeneration. In the zebrafish model of Duchenne muscular dystrophy (DMD), loss of sarcolemmal integrity precedes the detachment of muscle fibers from the basement membrane at the MTJ [6]. Increased levels of MMP-9 are associated with the pathogenesis of DMD in the $m d x$ mouse model, the canine X-linked muscular dystrophy in Japan $\left(\mathrm{CXMD}_{\mathrm{J}}\right)$ model, and muscle biopsies from human DMD patients [14,39,40]. The laminin $\alpha 1$ chain of the laminin 111 protein promotes the production and activity of MMP-9 [41,42]. As increased MMP-9 levels are associated with the pathogenesis of DMD, and laminin 111 can stimulate the expression of MMP-9, we asked whether MMP-9 expression was increased in spns1 ${ }^{-1-}$ mutants. MMP-9 was expressed in wild-type embryos and spns $1^{-/-}$mutants at $24 \mathrm{hpf}$ (Figure 1(C1-D2)), but was not expressed in wild-type larvae at $3.5 \mathrm{dpf}$ (Figure 7A-A3). Transcription of $m m p 9$ was upregulated 15.1 fold in spns $1^{-1-}$ mutants compared to WT controls at $3.5 \mathrm{dpf}$ (Figure 7C, Table 3). This result was corroborated at the protein level: MMP-9 was readily detected in spns $1^{-1-}$ mutants at $3.5 \mathrm{dpf}$ but not in wild-type larvae (Figure 7B-B3 vs. Figure 7A-A3). Relative MMP-9 staining was significantly stronger in spns $1^{-1-}$ mutants compared with WT controls at $3.5 \mathrm{dpf}(p$-value $<0.01)$ (Figure 7D). These data indicate that MMP-9 was increased at the transcription and protein levels in spns1 ${ }^{-1-}$ muscle at a time when MMP-9 was normally not expressed.

Table 3. Quantitative Real-Time PCR Results.

\begin{tabular}{ccc}
\hline Primer & $\Delta \Delta \mathbf{C q}$ & Exponential Change \\
\hline tweak & -5.09 & 34.12 \\
\hline$m m p 9$ & -3.92 & 15.12 \\
\hline
\end{tabular}

\subsection{Tnfsd12-Fn14 Signaling Axis Regulated Increased MMP-9 Expression}

Proinflammatory cytokines are known mediators of skeletal muscle degeneration. The muscle-wasting cytokine TNF superfamily member 12 (tnfsf12) induces MMP-9 expression in skeletal muscle. In MMP-9 knockout mice, tnfsf12-induced muscle degeneration in inhibited, indicating that MMP-9 is required for tnfsf12-induced muscle degeneration

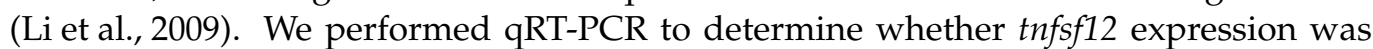
increased in spns $1^{-/}$mutants at $3.5 \mathrm{dpf}$. We found that transcription of $\operatorname{tnfsf} 12$ was upregulated 34.2 fold in spns1 ${ }^{-/-}$mutants compared to WT controls at $3.5 \mathrm{dpf}$ (data not shown). Next we asked whether Tnfsf12 induces $m m p 9$ gene expression in spns $1^{-/}$mutants. Tnfsf12 signaling involves the binding of Tnfsf12 to its receptor, fibroblast growth factorinducible 14 (Fn14) [43]. The small molecule inhibitor aurintricarboxylic acid (ATA) blocks Tnfsf12-induced signaling downstream from the Tnfsf12-Fn14 complex [43]. Although Tnfsf12-Fn14 signaling is not required for normal development in mice, we administered ATA beginning at $2 \mathrm{dpf}$ to avoid any potential disruption of muscle development (Maecker et al., 2005). We treated embryos continuously with $100 \mu \mathrm{M}$ ATA (with $0.1 \% \mathrm{EtOH}$ ) beginning at $2 \mathrm{dpf}$ and analyzed MMP-9 expression at $3.5 \mathrm{dpf}$. This dose was based on a previous zebrafish study showing that $100 \mu \mathrm{M}$ ATA caused no significant damage to embryos, while concentrations above $100 \mu \mathrm{M}$ ATA caused embryo death [22]. Controls were treated with $0.1 \% \mathrm{EtOH}$. MMP-9 was not detected in WT controls treated with $0.1 \% \mathrm{EtOH}$ or $100 \mu \mathrm{M}$ ATA (Figure 8A,B). MMP-9 staining was detected in spns $1^{-/}$mutants treated with $0.1 \% \mathrm{EtOH}$; however, MMP-9 staining was not readily visible in ATA treated spns1 ${ }^{-1-}$ mutants (Figure 8C,D). 

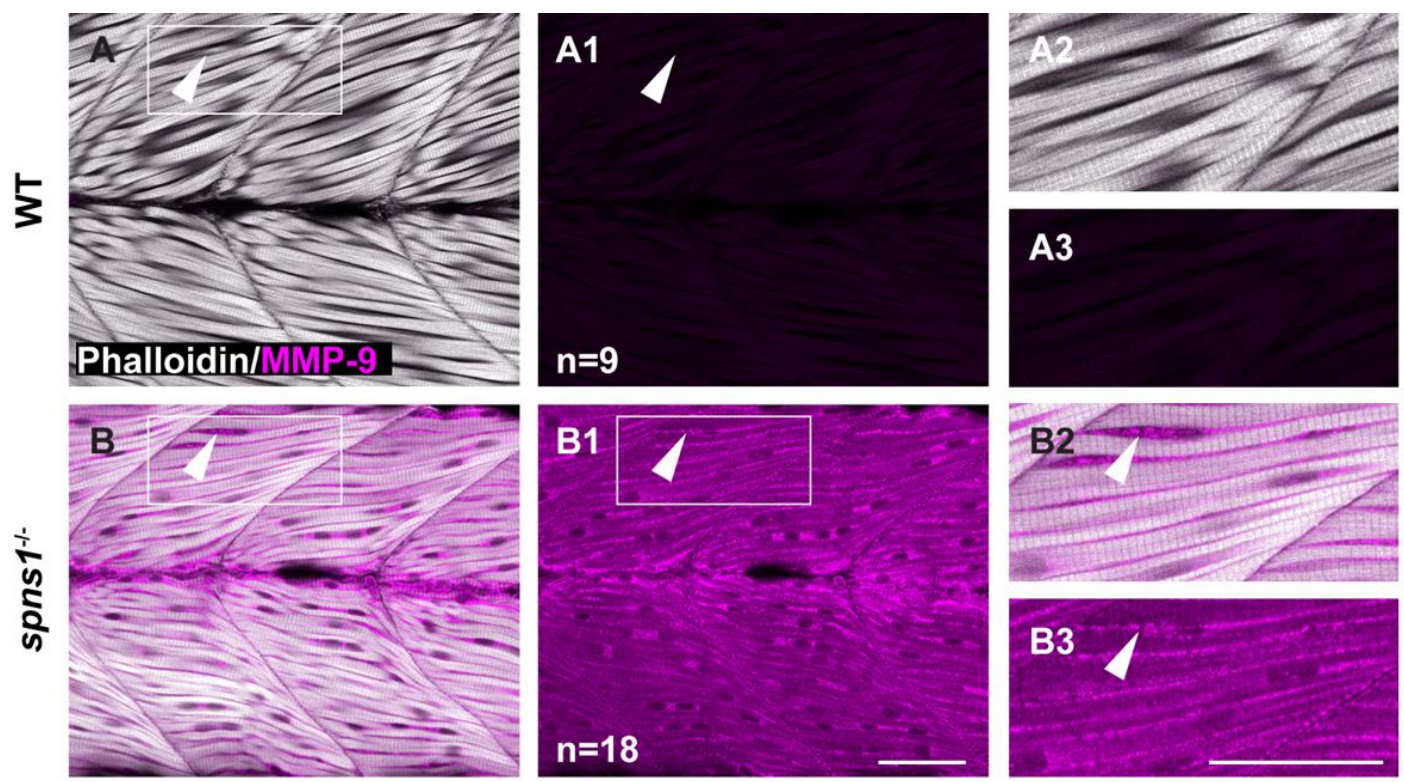

C: mRNA expression relative to control

D: Relative anti-MMP-9

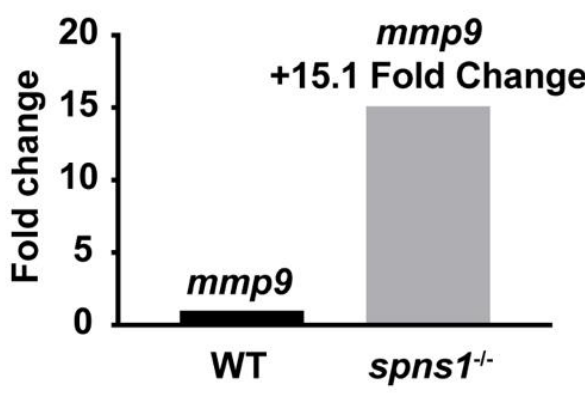
staining intensity at the NMJ

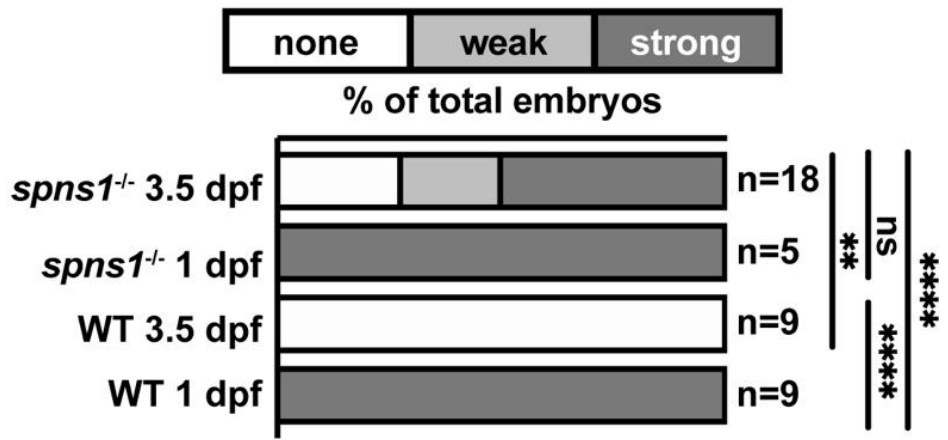

Figure 7. $m m p 9$ mRNA and protein are upregulated in spns1-/- larvae at $3.5 \mathrm{dpf}$. (A,B) Anterior left, dorsal top, side-mounted larvae. Phalloidin (white) to visualize actin and MMP-9 antibody staining (pseudo-colored fuchsia). (A,A2,B,B2) Merged phalloidin and MMP-9 channels and (A1,B1,A3,B3) MMP-9 single channel. (A1) MMP-9 is not detected in WT muscle. (B1) MMP-9 is detected in spns1 $1^{-/}$muscle. White arrowheads highlight MMP-9 staining (or lack thereof in wild-type larvae). (C) Relative mmp9 mRNA expression in WT and spns1 ${ }^{-1}$ larvae measured as fold change in $3.5 \mathrm{dpf}$ larvae. (D) Percentage of spns $1^{-/-}$and WT at 1 and $3.5 \mathrm{dpf}$ with qualitatively scored relative fluorescence intensity. ${ }^{* *} p<0.01,{ }^{* * * *} p<0.0001$. Scale bars $50 \mu \mathrm{m}$. 

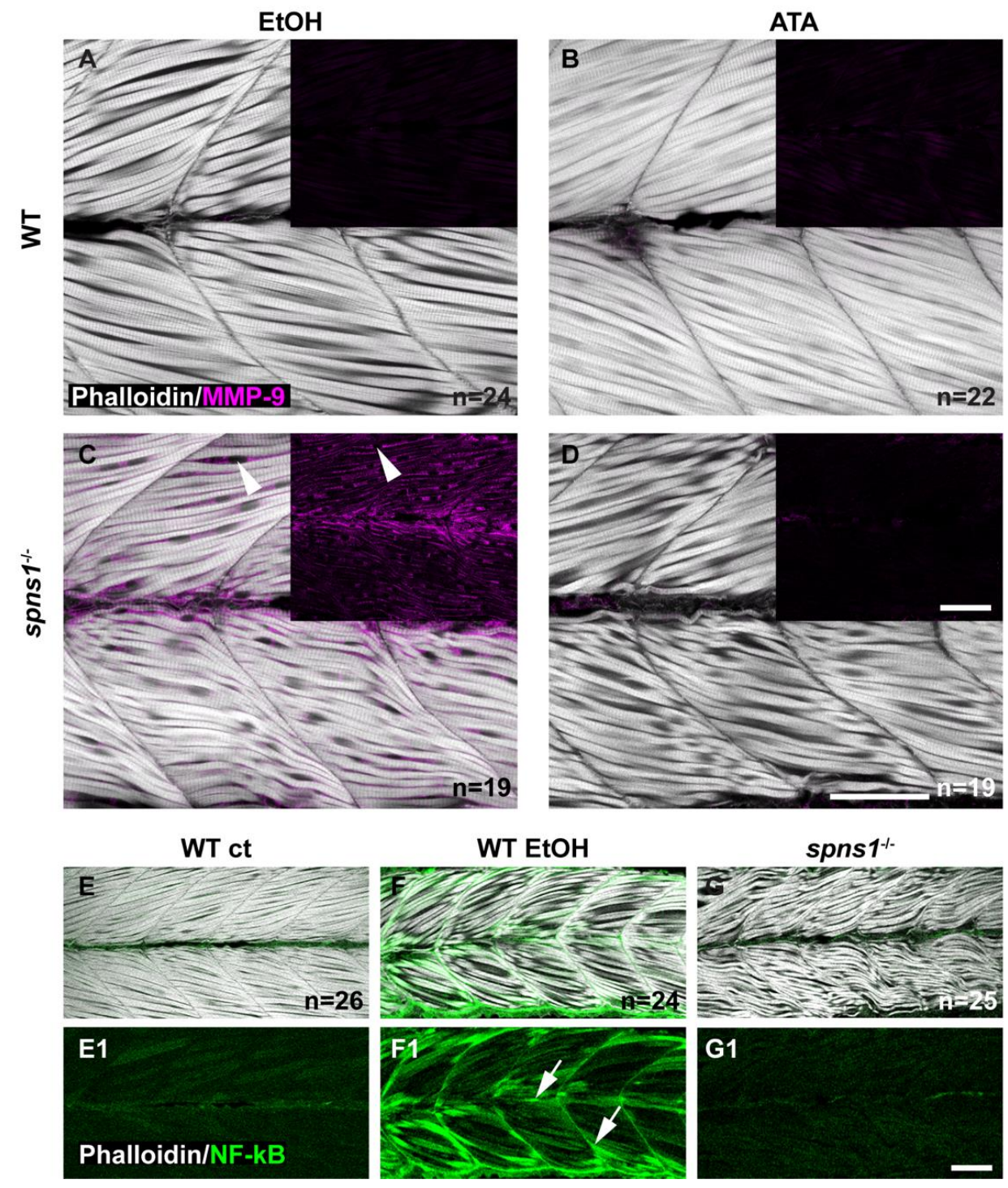

\section{$\mathrm{H}$ : Mean grey value at $3 \mathrm{dpf}$}

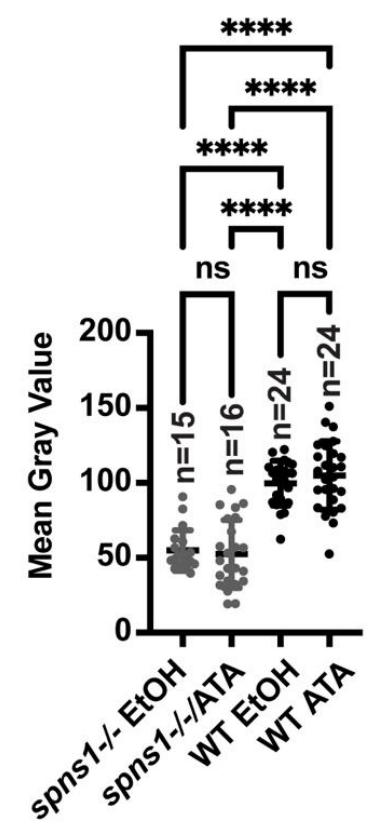

\section{I: Muscle damage severity}

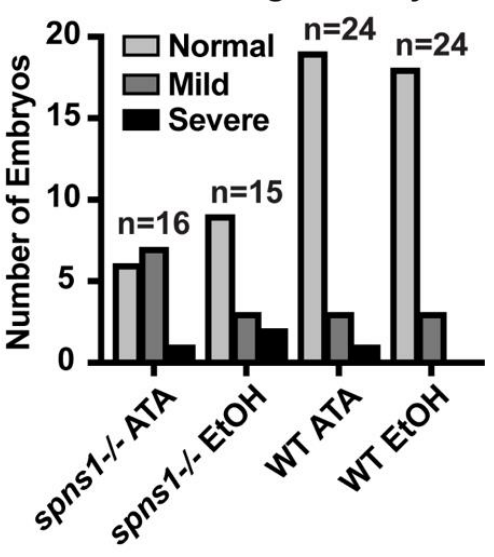

Figure 8. Tnfsf12 inhibition affects MMP-9 protein levels independently of NF- $\kappa B$ but reducing MMP-9 does not reduce muscle degeneration. (A-G) Anterior left, dorsal top, side-mounted 3.5 dpf larvae. (A-D) Merged phalloidin and MMP-9 stained (pseudo-colored fuchsia) channels in WT and spns1-/- larvae, MMP-9 staining alone is in black inset boxes. Zebrafish were treated with either $2 \%$ EtOH from $30 \mathrm{hpf}-3.5 \mathrm{dpf}$ or $100 \mu \mathrm{M}$ TWEAK inhibitor Aurintricarboxylic acid (ATA) from 30 hpf-3.5 dpf. (A,B) Absence of MMP-9 protein in WT zebrafish treated with EtOH and with ATA. (C) Presence of MMP-9 protein in spns1 ${ }^{-/}$zebrafish treated with EtOH. White arrowheads indicate MMP-9 positive staining. (D) Absence of MMP-9 protein in spns1 $1^{-/-}$zebrafish treated with ATA. (E-G) Merged phalloidin (white) and EGFP (green) channels in EtOH treated and untreated $T g(N F-\kappa B-E G F P) ; \mathrm{WT}$ and $T g(N F-\kappa B-E G F P) ; s p n s 1^{-/}$zebrafish. (E1-G1) EGFP single channel. White arrows indicate $N F-\kappa B-E G F P$ positive muscle fibers in (F1) EtOH treated WT zebrafish, but $N F-\kappa B-E G F P$ is not upregulated in spns1 $1^{-/}$mutants. (H) Changes in muscle structure, as measured by mean gray value, were insignificant in $3 \mathrm{dpf}$ spns1 ${ }^{-/-}$zebrafish treated with ATA or EtOH vehicle control. (I) Treatment with ATA and the subsequent reduction in MMP-9 does not improve muscle structure in spns1 ${ }^{-/}$mutants as assayed by either birefringence $(\mathbf{H})$ or qualitative assessment of muscle structure after phalloidin staining (I). Categories are normal (healthy tissue), mild (fiber disorganization), or severe (fiber disorganization and fiber balls/MTJ failure). ${ }^{* * *} p<0.0001$. Scale bars $50 \mu \mathrm{m}$.

Tnfsf12 induces gene expression of mmp9 via the activation of NF- $\kappa$ B and AP-1 transcription factors in skeletal muscle [44]. Thus, we used the $\operatorname{Tg}(N F-\kappa B: E G F P)$ zebrafish line to ask whether Tnfsf12-induced activation of NF- $\kappa$ B is detected in $s p n s 1^{-/-}$mutants [18]. The $\mathrm{Tg}(\mathrm{NF}-\kappa \mathrm{B}: E G F P)$ line was crossed into the $s p n s 1^{+/-}$line to detect NF- $\kappa \mathrm{B}$ activity in spns1 ${ }^{-/-}$ mutants. Previous work from our lab showed that zebrafish continuously exposed to 
$2 \% \mathrm{EtOH}$ from $30 \mathrm{hpf}-3 \mathrm{dpf}$ exhibit increased NF- $\kappa \mathrm{B}$ activity as evidenced by increased GFP expression in muscle fibers [45]. Hence, we used WT embryos exposed to $2 \% \mathrm{EtOH}$ from

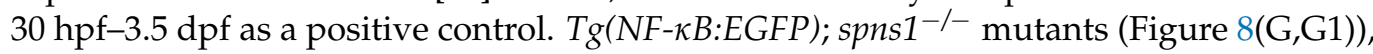
$T g(N F-\kappa B: E G F P)$; WT negative controls (Figure 8(E,E1)), and Tg(NF- $\kappa B: E G F P) ;$ WT positive controls (Figure 8(F,F1)) were fixed at $3.5 \mathrm{dpf}$. Muscle fibers in $T g(N F-\kappa B: E G F P)$; WT negative controls expressed significantly less GFP at $3.5 \mathrm{dpf}$ compared with muscle fibers in $T g(N F-\kappa B: E G F P)$; WT positive controls ( $p$-value < 0.0001) (Figure 8(E1,F1)). Muscle fibers in $T g(N F-\kappa B: E G F P) ; s p n s 1^{-/-}$mutants also expressed significantly less GFP at $3.5 \mathrm{dpf}$ compared with muscle fibers in $T g(N F-\kappa B: E G F P)$; WT positive controls $(p$-value $<0.0001)$ (Figure $8(\mathrm{G} 1, \mathrm{~F} 1)$ ). There was no significant difference of GFP expression in muscle fibers between the $T g(N F-\kappa B: E G F P)$; spns $1^{-/-}$mutants and $T g(N F-\kappa B: E G F P)$; WT negative controls $(p$-value $=0.5059)($ Figure $8(\mathrm{G} 1, \mathrm{E} 1))$. These data suggest that Tnfsf12 induced gene expression of $m m p 9$ independent of NF- $\kappa$ B activation in spns $1^{-/-}$mutants.

\subsection{Reduced MMP-9 Expression Did Not Impact Muscle Degeneration}

Increased MMP-9 levels are associated with pathogenesis in DMD [14,39,40]. The above data indicate that ATA treatment dramatically reduces MMP-9 levels in spns1 ${ }^{-1-}$ mutants. We thus asked whether reducing MMP-9 levels would improve muscle structure in spns $1^{-/}$mutants. We assessed muscle structure by quantifying birefringence in spns $1^{-1-}$ mutants. Healthy muscle is an organized structure consisting of attached, parallel muscle fibers which act as a light polarizer. By passing the light through a polarizing filter, a comparison can be made between organized muscle, appearing bright white and damaged and/or disorganized muscle, which appears dark. This difference in order, as measured by birefringence, can be quantified by measuring average mean gray values. We found that there was no significant difference in birefringence (measured as mean gray value) (Figure $8 \mathrm{H}$ ) in control versus ATA treatment. There was no obvious difference between the number of zebrafish that had normal, mild, or severe muscle damage (Figure 8I) in ATA versus ethanol treated embryos as measured by phalloidin staining of the muscle fibers.

\section{Discussion}

Modulation of protein turnover is important for muscle development and homeostasis. Protein turnover requires a balance between the rate of protein synthesis and the rate of protein degradation. The efficiency of protein degradation is dependent upon regulation of lysosomal $\mathrm{pH}$. Our data support the hypothesis that dysregulation of lysosomal $\mathrm{pH}$ contributes to muscle degeneration. It is known that loss of the carbohydrate $/ \mathrm{H}^{+} \mathrm{sym}-$ porter spinster (spns1) results in premature senescence [12,16,25], but it was not known whether muscle develops normally prior to muscle degeneration. Here, we show that loss of spns1 does not affect skeletal muscle development. Muscle degeneration begins approximately $3.5 \mathrm{dpf}$. At this time, there was misregulation of the extracellular matrix at the myotendinous junction (MTJ). Expression of the developmental isoform of laminin, laminin 111, was observed at MTJs in spns $1^{-/-}$mutant embryos. This reexpression of laminin 111 appeared to be a compensatory response, as disruption of the laminin receptor Itga7 exacerbated the phenotype in spns $1^{-/-}$mutant embryos. However, re-expression of laminin 111 was not sufficient for muscle homeostasis in spns $1^{-/}$mutant embryos. We hypothesize that this is mainly due to the fact that the main mechanism underlying muscle degeneration in spns1 $1^{-/}$mutants is membrane instability, which is not improved with increased cell-matrix adhesion. Similar to other degenerative contexts where membrane stability is compromised, MMP-9 expression was upregulated in spns $1^{-/-}$mutant embryos. In contrast to these other contexts, inhibition of MMP-9 expression did not reduce muscle degeneration (summarized in Table 4). Together, these data indicate that dysregulated lysosomal $\mathrm{pH}$ has pleiotropic effects on zebrafish skeletal muscle, resulting in muscle degeneration. 
Table 4. Comparison of $3.5 \mathrm{dpf}$ to $1 \mathrm{dpf}$ wildtype, spns1-/-, dag1; spns1-/-, and itga7; spns1-/- zebrafish.

\begin{tabular}{|c|c|c|c|c|}
\hline Marker Being Assessed & $\begin{array}{l}\text { Wildtype (WT) } \\
3.5 \mathrm{dpf}\end{array}$ & $\begin{array}{l}\text { spns } 1^{-1-} \\
3.5 \mathrm{dpf}\end{array}$ & $\begin{array}{l}\text { dag1; spns1 } 1^{-1-} \\
3.5 \mathrm{dpf}\end{array}$ & $\begin{array}{c}\text { itga7; spns } 1^{-1-} \\
3.5 \mathrm{dpf}\end{array}$ \\
\hline Lysotracker & No puncta & Positive puncta & N/A & $\mathrm{N} / \mathrm{A}$ \\
\hline MMP9 mRNA & $\mathrm{N} / \mathrm{A}$ & +15.1 fold compared to WT & $\mathrm{N} / \mathrm{A}$ & $\mathrm{N} / \mathrm{A}$ \\
\hline $\begin{array}{l}\text { MMP9 antibody staining } \\
\text { compared to } 1 \mathrm{dpf}\end{array}$ & $\begin{array}{c}\text { MMP9 absent compared to } 1 \\
\text { dpf WT } \\
p<0.0001\end{array}$ & $\begin{array}{l}\text { MMP9 returns to } 1 \mathrm{dpf} \\
\text { spns } 1^{-/-} \text {level }\end{array}$ & $\mathrm{N} / \mathrm{A}$ & $\mathrm{N} / \mathrm{A}$ \\
\hline $\begin{array}{l}\text { MMP9 antibody staining } \\
\text { after } 100 \mu \mathrm{M} \\
\text { ATA (Tnfsf12 inhibitor) }\end{array}$ & $\begin{array}{l}\text { MMP9 absent in ATA and in } \\
0.1 \% \text { EtOH vehicle control } \\
\text { treated embryos }\end{array}$ & $\begin{array}{l}\text { MMP9 absent in ATA but } \\
\text { present in } 0.1 \% \text { EtOH } \\
\text { vehicle control treated } \\
\text { embryos }\end{array}$ & $\mathrm{N} / \mathrm{A}$ & $\mathrm{N} / \mathrm{A}$ \\
\hline$N F-\kappa B: E G F P$ relative levels & $\begin{array}{c}\text { Lower in negative controls } \\
\text { than EtOH treated } \\
p \text {-value }<0.0001\end{array}$ & $\begin{array}{l}\text { Less than positive controls } \\
\text { same as negative controls } \\
p \text {-values }<0.0001\end{array}$ & $\mathrm{~N} / \mathrm{A}$ & $\mathrm{N} / \mathrm{A}$ \\
\hline $\begin{array}{l}\text { Sarcolemma instability: } \\
\text { beta-dystroglycan (b-DG) } \\
\text { at the myotendinous } \\
\text { junction(MTJ) versus } \\
\text { at fiber ends }\end{array}$ & $\mathrm{N} / \mathrm{A}$ & $\begin{array}{c}\text { More embryos with } \\
\text { b-DG at MTJ } p \text {-value }<0.001 \\
\text { More segments with } \\
\text { MTJ staining } \\
p \text {-value }<0.0001\end{array}$ & $\mathrm{~N} / \mathrm{A}$ & $\mathrm{N} / \mathrm{A}$ \\
\hline $\begin{array}{l}\text { Laminin-111 staining at } \\
3.5 \mathrm{dpf} \text { compared to } 1 \mathrm{dpf}\end{array}$ & $\begin{array}{c}\text { Laminin-111 absent } \\
\text { compared to } 1 \mathrm{dpf} \text { WT } \\
p<0.0001\end{array}$ & $\begin{array}{c}\text { Laminin- } 111 \text { returns to } \\
1 \mathrm{dpf} s p n s^{-/-} \text {level } \\
\text { NS } \\
p \text {-value }>0.9999\end{array}$ & $\begin{array}{c}\text { Laminin-111 } \\
\text { Expression returns to } \\
1 \mathrm{dpf} \text { dag1; spns1 } 1^{-/-} \\
\text {level } \\
\mathrm{NS} \\
p \text {-value }=0.057\end{array}$ & $\begin{array}{c}\text { Laminin-111 } \\
\text { expression returns to } \\
1 \mathrm{dpf} \text { itga7; spns1-/- } \\
\text { level } \\
\text { NS }\end{array}$ \\
\hline $\begin{array}{l}\text { Laminin-111 staining at } \\
3.5 \mathrm{dpf} \text { after } 50 \mu \mathrm{M} \\
\text { cyclopamine (sonic } \\
\text { hedgehog signaling blocker) }\end{array}$ & $\begin{array}{l}\text { Stronger compared to WT } \\
\text { vehicle controls } \\
p \text {-value }<0.0001\end{array}$ & $\begin{array}{c}\text { No change compared to } \\
\text { spns1-/- vehicle controls } \\
\text { NS } \\
p \text {-value }=0.1838\end{array}$ & $\mathrm{~N} / \mathrm{A}$ & $\mathrm{N} / \mathrm{A}$ \\
\hline
\end{tabular}

Although it has been shown that increased SA-beta-galactosidase activity, a marker of senescence, is detected in spns $1^{-/}$zebrafish at $3.5 \mathrm{dpf}$ [16], it was not known if spns1 is required for initial muscle development. We found that initial muscle development proceeds normally in spns1 ${ }^{-/-}$mutants prior to overt muscle degeneration at $3.5 \mathrm{dpf}$. This result suggests that dysregulated lysosomal $\mathrm{pH}$ causes muscle damage following primary muscle development in zebrafish and contributes to the growing body of evidence indicating that proper lysosomal function is critical for muscle homeostasis. Dysregulation of lysosomal $\mathrm{pH}$ has been shown to cause muscle atrophy induced by the antimalarial drug Chloroquine [46]. Loss of cathepsin D-mediated lysosomal protein degradation, which requires a $\mathrm{pH}$ of 4.5-5, has also been linked to muscle atrophy in both human patients and zebrafish [47,48]. Although there is no known human disease linked to spns1, Cathepsin $\mathrm{D}$ acts downstream of Spns1: loss of spns1 contributes to impaired cathepsin D activity and increased spns1 promotes cathepsin D activity [13,49]. Interestingly, a previous study showed that $\mathrm{EtOH}$ treatment increases lysosomal $\mathrm{pH}$ in rat hepatocytes, thereby causing significantly reduced protein degradation [50]. We recently found that ethanol exposure causes muscle damage in zebrafish [45]. It will be interesting in the future to determine if dysregulated lysosomal $\mathrm{pH}$ contributes to $\mathrm{EtOH}$-induced muscle degeneration in zebrafish.

Interactions between muscle cells and their surrounding ECM regulate cellular adaptive responses in homeostasis and disease. Laminin 111 is a principal ECM component that is required for the formation of normal muscle and MTJs during embryonic development [30,51]. After initial muscle development, laminin 111 is replaced with laminin 211 [52]. MDC1A is a muscular dystrophy caused by mutations in lama2 but laminin-111 protein therapy significantly improves pathology of mouse models for MDC1A [53]. We hypothesized that ectopic laminin 111 expression in spns1 ${ }^{-1-}$ larvae at $3.5 \mathrm{dpf}$ was an innate adaptive response to muscle degeneration. One direct approach to test this hypothesis would be to generate laminin apha1, beta1, or gamma1 spns1 $1^{-1-}$ double mutants. 
However, since laminin 111 is required for normal muscle development [30,51], we tested our hypothesis with Integrin $\alpha 7$ mutants because Itg $\alpha 7$ is not required for initial muscle development. After using CRISPR-Cas9 to make Integrin $\alpha 7$ mutants, we then generated itga7; spns $1^{-/}$double mutants and observed increased muscle degeneration compared to single spns $1^{-/-}$mutant embryos.

Matrix metalloproteinases (MMPs), a group of zinc-dependent endopeptidases, are known to cleave all ECM proteins. MMP gene expression and activity is tightly regulated during development and homeostasis; however, changes in MMP expression and activity occur in many diseases [54]. With respect to muscle diseases, it has been shown that MMP-9 plays an important role in the pathogenesis of DMD [39,55]. Given that both spns1 ${ }^{-1-}$ larvae and the zebrafish model of DMD show muscle damage that results from sarcolemmal instability, we asked whether MMP-9 might participate in muscle degeneration induced by loss of spns1. MMP-9 mRNA and protein levels were increased in spns1 ${ }^{-1-}$ larvae compared to WT controls. The cytokine Tnfsf12 has been shown to promote MMP-9 expression and muscle damage in multiple forms of muscle atrophy [56-58]. Our data suggest that Tnfsf12 expression mediates increased MMP-9 expression in spns $1^{-/-}$larvae because treatment with ATA dramatically decreases MMP-9 expression in spns1 $1^{-/}$larvae. However, treatment with ATA was not sufficient to reduce muscle damage in spns1 ${ }^{-1-}$ larvae. This result suggests that in contrast to DMD, increased MMP-9 is not a major mechanism underlying muscle degeneration in spns $1^{-/-}$larvae. It will be interesting in the future to determine the mechanisms underlying loss of sarcolemmal integrity in zebrafish in response to dysregulated lysosomal $\mathrm{pH}$.

We have addressed the impact of dysregulated lysosomal $\mathrm{pH}$ on muscle development in zebrafish. Our data showed that spns1 $1^{-1-}$ larvae display normal initial muscle development, which was followed by rapid loss of muscle integrity and increased expression of the ECM protein laminin 111. Increased laminin 111 appeared to be compensatory because disruption of adhesion to laminin increased muscle degeneration in spns1 $1^{-1-}$ larvae. Finally, spns $1^{-/-}$larvae exhibit muscle damage caused by sarcolemmal instability that coincides with Tnfsf12-induced MMP-9 expression, but inhibition of Tnfsf12 signaling was not sufficient to maintain muscle integrity in spns1 $1^{-/}$larvae. These data provide novel insight into the consequences of dysregulated lysosomal $\mathrm{pH}$ on muscle development. Although further studies are required to show whether dysregulated lysosomal $\mathrm{pH}$ impacts homeostasis in mammals, our data set the stage for these future experiments.

Author Contributions: Conceptualization, E.C.C. and C.A.H.; methodology, E.C.C.; validation, E.C.C., M.A. and C.S.; formal analysis, E.C.C., M.A., C.S. and C.A.H.; investigation, E.C.C., M.A., S.S.A. and C.S.; resources, C.A.H.; data curation, E.C.C. and C.A.H.; writing-original draft preparation, E.C.C.; writing-review and editing, E.C.C., M.A., C.S., C.A.H.; visualization, E.C.C. and C.S.; supervision, C.A.H.; project administration, C.A.H.; funding acquisition, C.A.H. All authors have read and agreed to the published version of the manuscript.

Funding: This research was funded by a University of Maine Seed Grant, NSF MRI (1726541), and NIH RO1 (AR075836-01).

Institutional Review Board Statement: The study was conducted according to the guidelines of the Declaration of Helsinki, and approved by the Institutional Animal Care and Use Committee at the University of Maine, Orono, ME 04469, USA (Protocol approval not required for fish $\leq 3 \mathrm{dpf}$ ).

Data Availability Statement: All data is contained within the article.

Acknowledgments: Mark Nilan for exceptional zebrafish care at the UMaine Zebrafish Facility.

Conflicts of Interest: The authors declare no conflict of interests. The funders had no role in the design of the study; in the collection, analyses, or interpretation of data; in the writing of the manuscript, or in the decision to publish the results. 


\section{References}

1. Tesseraud, S.; Chagneau, A.M.; Grizard, J. Muscle Protein Turnover during Early Development in Chickens Divergently Selected for Growth Rate. Poult. Sci. 2000, 79, 1465-1471. [CrossRef] [PubMed]

2. Nemova, N.N.; Lysenko, L.A.; Kantserova, N.P. [Degradation of skeletal muscle protein during growth and development of salmonid fish]. Ontogenez 2016, 47, 197-208. [CrossRef]

3. Rajawat, Y.S.; Hilioti, Z.; Bossis, I. Aging: Central Role for Autophagy and the Lysosomal Degradative System. Ageing Res. Rev. 2009, 8, 199-213. [CrossRef] [PubMed]

4. Costa, M.L.; Escaleira, R.C.; Jazenko, F.; Mermelstein, C.S. Cell Adhesion in Zebrafish Myogenesis: Distribution of Intermediate Filaments, Microfilaments, Intracellular Adhesion Structures and Extracellular Matrix. Cell Motil. 2008, 65, 801-815. [CrossRef] [PubMed]

5. Gullberg, D.; Velling, T.; Sjöberg, G.; Salmivirta, K.; Gaggero, B.; Tiger, C.F.; Edström, L.; Sejersen, T. Tenascin-C Expression Correlates with Macrophage Invasion in Duchenne Muscular Dystrophy and in Myositis. Neuromuscul. Disord. 1997, 7, 39-54. [CrossRef]

6. Hall, T.E.; Bryson-Richardson, R.J.; Berger, S.; Jacoby, A.S.; Cole, N.J.; Hollway, G.E.; Berger, J.; Currie, P.D. The Zebrafish Candyfloss Mutant Implicates Extracellular Matrix Adhesion Failure in Laminin A2-Deficient Congenital Muscular Dystrophy. Proc. Natl. Acad. Sci. USA 2007, 104, 7092-7097. [CrossRef]

7. Leonoudakis, D.; Huang, G.; Akhavan, A.; Fata, J.E.; Singh, M.; Gray, J.W.; Muschler, J.L. Endocytic Trafficking of Laminin Is Controlled by Dystroglycan and Is Disrupted in Cancers. J. Cell Sci. 2014, 127, 4894-4903. [CrossRef]

8. Carmignac, V.; Svensson, M.; Körner, Z.; Elowsson, L.; Matsumura, C.; Gawlik, K.I.; Allamand, V.; Durbeej, M. Autophagy Is Increased in Laminin A2 Chain-Deficient Muscle and Its Inhibition Improves Muscle Morphology in a Mouse Model of MDC1A. Hum. Mol. Genet. 2011, 20, 4891-4902. [CrossRef]

9. Grumati, P.; Coletto, L.; Sabatelli, P.; Cescon, M.; Angelin, A.; Bertaggia, E.; Blaauw, B.; Urciuolo, A.; Tiepolo, T.; Merlini, L.; et al. Autophagy Is Defective in Collagen VI Muscular Dystrophies, and Its Reactivation Rescues Myofiber Degeneration. Nat. Med. 2010, 16, 1313-1320. [CrossRef]

10. Graves, A.R.; Curran, P.K.; Smith, C.L.; Mindell, J.A. The Cl-/H+ Antiporter ClC-7 Is the Primary Chloride Permeation Pathway in Lysosomes. Nature 2008, 453, 788-792. [CrossRef] [PubMed]

11. Sagné, C.; Agulhon, C.; Ravassard, P.; Darmon, M.; Hamon, M.; El Mestikawy, S.; Gasnier, B.; Giros, B. Identification and Characterization of a Lysosomal Transporter for Small Neutral Amino Acids. Proc. Natl. Acad. Sci. USA 2001, 98, 7206-7211. [CrossRef] [PubMed]

12. Sasaki, T.; Lian, S.; Khan, A.; Llop, J.R.; Samuelson, A.V.; Chen, W.; Klionsky, D.J.; Kishi, S. Autolysosome Biogenesis and Developmental Senescence Are Regulated by Both Spns1 and V-ATPase. Autophagy 2017, 13, 386-403. [CrossRef] [PubMed]

13. Rong, Y.; McPhee, C.K.; McPhee, C.; Deng, S.; Huang, L.; Chen, L.; Liu, M.; Tracy, K.; Baehrecke, E.H.; Baehreck, E.H.; et al. Spinster Is Required for Autophagic Lysosome Reformation and MTOR Reactivation Following Starvation. Proc. Natl. Acad. Sci. USA 2011, 108, 7826-7831. [CrossRef] [PubMed]

14. Nadarajah, V.D.; van Putten, M.; Chaouch, A.; Garrood, P.; Straub, V.; Lochmüller, H.; Ginjaar, H.B.; Aartsma-Rus, A.M.; van Ommen, G.J.B.; den Dunnen, J.T.; et al. Serum Matrix Metalloproteinase-9 (MMP-9) as a Biomarker for Monitoring Disease Progression in Duchenne Muscular Dystrophy (DMD). Neuromuscul. Disord. 2011, 21, 569-578. [CrossRef] [PubMed]

15. Shiba, N.; Miyazaki, D.; Yoshizawa, T.; Fukushima, K.; Shiba, Y.; Inaba, Y.; Imamura, M.; Takeda, S.; Koike, K.; Nakamura, A. Differential Roles of MMP-9 in Early and Late Stages of Dystrophic Muscles in a Mouse Model of Duchenne Muscular Dystrophy. Biochim. Biophys. Acta 2015, 1852, 2170-2182. [CrossRef]

16. Kishi, S.; Bayliss, P.E.; Uchiyama, J.; Koshimizu, E.; Qi, J.; Nanjappa, P.; Imamura, S.; Islam, A.; Neuberg, D.; Amsterdam, A.; et al. The Identification of Zebrafish Mutants Showing Alterations in Senescence-Associated Biomarkers. PLoS Genet. 2008,4 , e1000152. [CrossRef]

17. Lin, Y.-Y.; White, R.J.; Torelli, S.; Cirak, S.; Muntoni, F.; Stemple, D.L. Zebrafish Fukutin Family Proteins Link the Unfolded Protein Response with Dystroglycanopathies. Hum. Mol. Genet. 2011, 20, 1763-1775. [CrossRef] [PubMed]

18. Kanther, M.; Sun, X.; Mühlbauer, M.; Mackey, L.C.; Flynn, E.J.; Bagnat, M.; Jobin, C.; Rawls, J.F. Microbial Colonization Induces Dynamic Temporal and Spatial Patterns of NF-KB Activation in the Zebrafish Digestive Tract. Gastroenterology 2011, 141, 197-207. [CrossRef]

19. Kimmel, C.B.; Ballard, W.W.; Kimmel, S.R.; Ullmann, B.; Schilling, T.F. Stages of Embryonic Development of the Zebrafish. Dev. Dyn. 1995, 203, 253-310. [CrossRef] [PubMed]

20. Gagnon, J.A.; Valen, E.; Thyme, S.B.; Huang, P.; Akhmetova, L.; Ahkmetova, L.; Pauli, A.; Montague, T.G.; Zimmerman, S.; Richter, C.; et al. Efficient Mutagenesis by Cas9 Protein-Mediated Oligonucleotide Insertion and Large-Scale Assessment of Single-Guide RNAs. PLoS ONE 2014, 9, e98186. [CrossRef] [PubMed]

21. Barresi, M.J.F.; Hutson, L.D.; Chien, C.-B.; Karlstrom, R.O. Hedgehog Regulated Slit Expression Determines Commissure and Glial Cell Position in the Zebrafish Forebrain. Development 2005, 132, 3643-3656. [CrossRef] [PubMed]

22. Kurusamy, S.; López-Maderuelo, D.; Little, R.; Cadagan, D.; Savage, A.M.; Ihugba, J.C.; Baggott, R.R.; Rowther, F.B.; MartínezMartínez, S.; Arco, P.G.-D.; et al. Selective Inhibition of Plasma Membrane Calcium ATPase 4 Improves Angiogenesis and Vascular Reperfusion. J. Mol. Cell Cardiol. 2017, 109, 38-47. [CrossRef] [PubMed] 
23. Berger, J.; Sztal, T.; Currie, P.D. Quantification of Birefringence Readily Measures the Level of Muscle Damage in Zebrafish. Biochem. Biophys. Res. Commun. 2012, 423, 785-788. [CrossRef] [PubMed]

24. Lee, F.H.F.; Zhang, H.; Jiang, A.; Zai, C.C.; Liu, F. Specific Alterations in Astrocyte Properties via the GluA2-GAPDH Complex Associated with Multiple Sclerosis. Sci Rep. 2018, 8, 12856. [CrossRef] [PubMed]

25. Sasaki, T.; Lian, S.; Qi, J.; Bayliss, P.E.; Carr, C.E.; Johnson, J.L.; Guha, S.; Kobler, P.; Catz, S.D.; Gill, M.; et al. Aberrant Autolysosomal Regulation Is Linked to The Induction of Embryonic Senescence: Differential Roles of Beclin 1 and P53 in Vertebrate Spns1 Deficiency. PLoS Genet. 2014, 10, e1004409. [CrossRef]

26. Khan, A.; Fahad, T.M.; Akther, T.; Zaman, T.; Hasan, M.F.; Islam Khan, M.R.; Islam, M.S.; Kishi, S. Carbofuran Accelerates the Cellular Senescence and Declines the Life Span of Spns1 Mutant Zebrafish. J. Cell Mol. Med. 2021, 25, 1048-1059. [CrossRef] [PubMed]

27. Talbot, J.; Maves, L. Skeletal Muscle Fiber Type: Using Insights from Muscle Developmental Biology to Dissect Targets for Susceptibility and Resistance to Muscle Disease. Wiley Interdiscip Rev. Dev. Biol. 2016, 5, 518-534. [CrossRef] [PubMed]

28. Jessen, J.R. Recent Advances in the Study of Zebrafish Extracellular Matrix Proteins. Dev. Biol. 2015, 401, 110-121. [CrossRef] [PubMed]

29. Peterson, M.T.; Henry, C.A. Hedgehog Signaling and Laminin Play Unique and Synergistic Roles in Muscle Development. Dev. Dyn. 2010, 239, 905-913. [CrossRef]

30. Anderson, C.; Thorsteinsdóttir, S.; Borycki, A.-G. Sonic Hedgehog-Dependent Synthesis of Laminin A1 Controls Basement Membrane Assembly in the Myotome. Development 2009, 136, 3495-3504. [CrossRef] [PubMed]

31. Chen, J.K.; Taipale, J.; Cooper, M.K.; Beachy, P.A. Inhibition of Hedgehog Signaling by Direct Binding of Cyclopamine to Smoothened. Genes Dev. 2002, 16, 2743-2748. [CrossRef]

32. Feng, X.; Adiarte, E.G.; Devoto, S.H. Hedgehog Acts Directly on the Zebrafish Dermomyotome to Promote Myogenic Differentiation. Dev. Biol. 2006, 300, 736-746. [CrossRef] [PubMed]

33. Hamill, K.J.; Kligys, K.; Hopkinson, S.B.; Jones, J.C.R. Laminin Deposition in the Extracellular Matrix: A Complex Picture Emerges. J. Cell Sci. 2009, 122, 4409-4417. [CrossRef] [PubMed]

34. Hayashi, Y.K.; Chou, F.L.; Engvall, E.; Ogawa, M.; Matsuda, C.; Hirabayashi, S.; Yokochi, K.; Ziober, B.L.; Kramer, R.H.; Kaufman, S.J.; et al. Mutations in the Integrin Alpha7 Gene Cause Congenital Myopathy. Nat. Genet. 1998, 19, 94-97. [CrossRef]

35. Yurchenco, P.D.; McKee, K.K.; Reinhard, J.R.; Rüegg, M.A. Laminin-Deficient Muscular Dystrophy: Molecular Pathogenesis and Structural Repair Strategies. Matrix Biol. 2018, 71-72, 174-187. [CrossRef] [PubMed]

36. Van Ry, P.M.; Minogue, P.; Hodges, B.L.; Burkin, D.J. Laminin-111 Improves Muscle Repair in a Mouse Model of Merosin-Deficient Congenital Muscular Dystrophy. Hum. Mol. Genet. 2014, 23, 383-396. [CrossRef]

37. Postel, R.; Vakeel, P.; Topczewski, J.; Knöll, R.; Bakkers, J. Zebrafish Integrin-Linked Kinase Is Required in Skeletal Muscles for Strengthening the Integrin-ECM Adhesion Complex. Dev. Biol. 2008, 318, 92-101. [CrossRef]

38. Bassett, D.I. Dystrophin Is Required for the Formation of Stable Muscle Attachments in the Zebrafish Embryo. Development 2003, 130, 5851-5860. [CrossRef]

39. Fukushima, K.; Nakamura, A.; Ueda, H.; Yuasa, K.; Yoshida, K.; Takeda, S.; Ikeda, S. Activation and Localization of Matrix Metalloproteinase-2 and -9 in the Skeletal Muscle of the Muscular Dystrophy Dog (CXMDJ). BMC Musculoskelet Disord. 2007, 8, 54. [CrossRef]

40. Kherif, S.; Lafuma, C.; Dehaupas, M.; Lachkar, S.; Fournier, J.G.; Verdière-Sahuqué, M.; Fardeau, M.; Alameddine, H.S. Expression of Matrix Metalloproteinases 2 and 9 in Regenerating Skeletal Muscle: A Study in Experimentally Injured and Mdx Muscles. Dev. Biol. 1999, 205, 158-170. [CrossRef]

41. Corcoran, M.L.; Kibbey, M.C.; Kleinman, H.K.; Wahl, L.M. Laminin SIKVAV Peptide Induction of Monocyte/Macrophage Prostaglandin E2 and Matrix Metalloproteinases. J. Biol. Chem. 1995, 270, 10365-10368. [CrossRef] [PubMed]

42. Kuratomi, Y.; Nomizu, M.; Tanaka, K.; Ponce, M.L.; Komiyama, S.; Kleinman, H.K.; Yamada, Y. Laminin Gamma 1 Chain Peptide, C-16 (KAFDITYVRLKF), Promotes Migration, MMP-9 Secretion, and Pulmonary Metastasis of B16-F10 Mouse Melanoma Cells. Br. J. Cancer 2002, 86, 1169-1173. [CrossRef] [PubMed]

43. Roos, A.; Dhruv, H.D.; Mathews, I.T.; Inge, L.J.; Tuncali, S.; Hartman, L.K.; Chow, D.; Millard, N.; Yin, H.H.; Kloss, J.; et al. Identification of Aurintricarboxylic Acid as a Selective Inhibitor of the TWEAK-Fn14 Signaling Pathway in Glioblastoma Cells. Oncotarget 2017, 8, 12234-12246. [CrossRef] [PubMed]

44. Li, H.; Mittal, A.; Paul, P.K.; Kumar, M.; Srivastava, D.S.; Tyagi, S.C.; Kumar, A. Tumor Necrosis Factor-Related Weak Inducer of Apoptosis Augments Matrix Metalloproteinase 9 (MMP-9) Production in Skeletal Muscle through the Activation of Nuclear Factor-KappaB-Inducing Kinase and P38 Mitogen-Activated Protein Kinase: A Potential Role of MMP-9 in Myopathy. J. Biol. Chem. 2009, 284, 4439-4450. [CrossRef] [PubMed]

45. Coffey, E.C.; Pasquarella, M.E.; Goody, M.F.; Henry, C.A. Ethanol Exposure Causes Muscle Degeneration in Zebrafish. J. Dev. Biol. 2018, 6, 7. [CrossRef] [PubMed]

46. Wibo, M.; Poole, B. Protein degradation in cultured cells. J.Cell Biol. 1974, 63, 430-440. [CrossRef] [PubMed]

47. Follo, C.; Ozzano, M.; Montalenti, C.; Santoro, M.M.; Isidoro, C. Knockdown of Cathepsin D in Zebrafish Fertilized Eggs Determines Congenital Myopathy. Biosci. Rep. 2013, 33, e00034. [CrossRef] 
48. Hersheson, J.; Burke, D.; Clayton, R.; Anderson, G.; Jacques, T.S.; Mills, P.; Wood, N.W.; Gissen, P.; Clayton, P.; Fearnley, J.; et al. Cathepsin D Deficiency Causes Juvenile-Onset Ataxia and Distinctive Muscle Pathology. Neurology 2014, 83, $1873-1875$. [CrossRef]

49. Yanagisawa, H.; Ishii, T.; Endo, K.; Kawakami, E.; Nagao, K.; Miyashita, T.; Akiyama, K.; Watabe, K.; Komatsu, M.; Yamamoto, D.; et al. L-Leucine and SPNS1 Coordinately Ameliorate Dysfunction of Autophagy in Mouse and Human Niemann-Pick Type C Disease. Sci. Rep. 2017, 7, 15944. [CrossRef]

50. Kharbanda, K.K.; McVicker, D.L.; Zetterman, R.K.; MacDonald, R.G.; Donohue, T.M. Flow Cytometric Analysis of Vesicular PH in Rat Hepatocytes after Ethanol Administration. Hepatology 1997, 26, 929-934. [CrossRef]

51. Gullberg, D.; Tiger, C.F.; Velling, T. Laminins during Muscle Development and in Muscular Dystrophies. Cell Mol. Life Sci. 1999, 56, 442-460. [CrossRef] [PubMed]

52. Goody, M.F.; Sher, R.B.; Henry, C.A. Hanging on for the Ride: Adhesion to the Extracellular Matrix Mediates Cellular Responses in Skeletal Muscle Morphogenesis and Disease. Dev. Biol. 2015, 401, 75-91. [CrossRef]

53. Rooney, J.E.; Knapp, J.R.; Hodges, B.L.; Wuebbles, R.D.; Burkin, D.J. Laminin-111 Protein Therapy Reduces Muscle Pathology and Improves Viability of a Mouse Model of Merosin-Deficient Congenital Muscular Dystrophy. Am. J. Pathol. 2012, 180, 1593-1602. [CrossRef]

54. Brkic, M.; Balusu, S.; Libert, C.; Vandenbroucke, R.E. Friends or Foes: Matrix Metalloproteinases and Their Multifaceted Roles in Neurodegenerative Diseases. Mediat. Inflamm. 2015, 2015, e620581. [CrossRef] [PubMed]

55. Dahiya, S.; Givvimani, S.; Bhatnagar, S.; Qipshidze, N.; Tyagi, S.C.; Kumar, A. Osteopontin-Stimulated Expression of Matrix Metalloproteinase-9 Causes Cardiomyopathy in the Mdx Model of Duchenne Muscular Dystrophy. J. Immunol. 2011, 187, 2723-2731. [CrossRef] [PubMed]

56. Dogra, C.; Changoua, H.; Wedhas, N.; Qin, X.; Wergedal, J.E.; Kumar, A. TNF-Related Weak Inducer of Apoptosis (TWEAK) Is a Potent Skeletal Muscle-Wasting Cytokine. FASEB J. 2007, 21, 1857-1869. [CrossRef]

57. Mittal, A.; Bhatnagar, S.; Kumar, A.; Lach-Trifilieff, E.; Wauters, S.; Li, H.; Makonchuk, D.Y.; Glass, D.J.; Kumar, A. The TWEAKFn14 System Is a Critical Regulator of Denervation-Induced Skeletal Muscle Atrophy in Mice. J. Cell Biol. 2010, 188, 833-849. [CrossRef]

58. Tajrishi, M.M.; Sato, S.; Shin, J.; Zheng, T.S.; Burkly, L.C.; Kumar, A. The TWEAK-Fn14 Dyad Is Involved in Age-Associated Pathological Changes in Skeletal Muscle. Biochem. Biophys. Res. Commun. 2014, 446, 1219-1224. [CrossRef] 